UDC 517.9

W. Czernous (Gdańsk Univ. Technology, Poland)

\title{
GENERALIZED SOLUTIONS OF MIXED PROBLEMS FOR FIRST-ORDER PARTIAL FUNCTIONAL DIFFERENTIAL EQUATIONS
}

\section{УЗАГАЛЬНЕНІ РОЗВ'ЯЗКИ МІШАНИХ ЗАДАЧ \\ ДЛЯ ФУНКЦІОНАЛЬНИХ ДИФЕРЕНЦІАЛЬНИХ РІВНЯНЬ З ЧАСТИННИМИ ПОХІДНИМИ ПЕРШОГО ПОРЯДКУ}

A theorem on the existence of solutions and their continuous dependence upon initial boundary conditions is proved. The method of bicaracteristics is used to transform the mixed problem into a system of integral functional equations of the Volterra type. The existence of solutions of this system is proved by a method of successive approximations and by using theorems on integral inequalities. Classical solutions of integral functional equations lead to generalized solutions of the original problem. Differential equations with deviated variables and differential integral problems can be obtained from a general model by specializing given operators.

Доведено теорему про існування розв'язків та їх неперервну залежність від початкових граничних умов. Для перетворення мішаної задачі у систему інтегральних функціональних рівнянь типу Вольтерра використано метод біхарактеристик. Існування розв'язків цієї системи доведено за допомогою методу послідовних наближень та теорем про інтегральні нерівності. Класичні розв' язки інтегральних функціональних рівнянь приводять до узагальнених розв' язків початкової задачі. Із загальної моделі за допомогою конкретизації заданих операторів можна отримати диференціальні рівняння із змінними, що відхиляються, та диференціальні інтегральні задачі.

1. Introduction. We formulate the functional differential problem. Let $a>0$, $h_{0} \in R_{+}, R_{+}=[0,+\infty), b=\left(b_{1}, \ldots, b_{n}\right) \in R^{n}$, and let $h=\left(h_{1}, \ldots, h_{n}\right) \in R_{+}^{n}$ be given, where $b_{i}>0$ for $1 \leq i \leq n$. We define the sets

$$
E=[0, a] \times[-b, b], \quad D=\left[-h_{0}, 0\right] \times[-h, h] .
$$

Let $\bar{c}=\left(c_{1}, \ldots, c_{n}\right)=b+h$ and

$$
\begin{gathered}
E_{0}=\left[-h_{0}, 0\right] \times[-\bar{c}, \bar{c}], \\
\partial_{0} E=[0, a] \times([-\bar{c}, \bar{c}] \backslash(-b, b)), \quad E^{*}=E_{0} \cup E \cup \partial_{0} E .
\end{gathered}
$$

Suppose that $z: E^{*} \rightarrow R$ and $(t, x) \in E$ are fixed. We define the function $z_{(t, x)}: D \rightarrow$ $\rightarrow R$ as follows:

$$
z_{(t, x)}(\tau, \xi)=z(t+\tau, x+\xi), \quad(\tau, \xi) \in D
$$

The function $z_{(t, x)}$ is the restriction of $z$ to the set $\left[t-h_{0}, t\right] \times[x-h, x+h]$ and this restriction is shifted to the set $D$. Elements of the space $C(D, R)$ will be denoted by $w, \bar{w}$ and so on. We denote by $\|\cdot\|_{0}$ the supremum norm in the space $C(D, R)$. Put $\Omega=E \times C(D, R) \times R^{n}$ and let

$$
\begin{gathered}
f: \Omega \rightarrow R, \quad \varphi: E_{0} \cup \partial_{0} E \rightarrow R, \\
\alpha_{0}:[0, a] \rightarrow R, \quad \alpha^{\prime}: E \rightarrow R^{n}, \quad \alpha^{\prime}=\left(\alpha_{1}, \ldots, \alpha_{n}\right),
\end{gathered}
$$

be given functions. We denote $\alpha(t, x)=\left(\alpha_{0}(t), \alpha^{\prime}(t, x)\right),(t, x) \in E$. We require that $\alpha(t, x) \in E$ for $(t, x) \in E$ and $\alpha_{0}(t) \leq t$ for $t \in[0, a]$. We will deal with the following mixed problem:

$$
\partial_{t} z(t, x)=f\left(t, x, z_{\alpha(t, x)}, \partial_{x} z(t, x)\right),
$$




$$
z(t, x)=\varphi(t, x) \text { on } \quad E_{0} \cup \partial_{0} E,
$$

where $\partial_{x} z=\left(\partial_{x_{1}} z, \ldots, \partial_{x_{n}} z\right)$.

A function $\tilde{z}:\left[-h_{0}, \xi\right] \times[-\bar{c}, \bar{c}] \rightarrow R$ where $0<\xi \leq a$, is a generalized solution of (1), (2) if it is continuous and

(i) the derivatives $\left(\partial_{x_{1}} \tilde{z}, \ldots, \partial_{x_{n}} \tilde{z}\right)=\partial_{x} \tilde{z}$ exist on $[0, \xi] \times[-b, b]$ and the function $\tilde{z}(\cdot, x):\left[-h_{0}, \xi\right] \rightarrow R$ is absolutely continuous on $[0, \xi]$ for each $x \in[-b, b]$;

(ii) for each $x \in[-b, b]$ equation (1) is satisfied for almost all $t \in[0, \xi]$ and condition (2) holds on $\left(E_{0} \cup \partial_{0} E\right) \times\left[-h_{0}, \xi\right] \times R^{n}$.

Note that our hereditary setting contains well-known delay structures as particular cases.

The simplest differential equation with deviated variables is obtained in the following way: put $h_{0}=0, h=0$, and suppose that $F: E \times R \times R^{n} \rightarrow R$ is a given function. Consider the operator $f$ defined by

$$
f(t, x, w, q)=F(t, x, w(0,0), q), \quad(t, x, w, q) \in \Omega .
$$

Then

$$
f\left(t, x, z_{\alpha(t, x)}, q\right)=F(t, x, z(\alpha(t, x)), q)
$$

and equation (1) is equivalent to the differential equation with deviated variables

$$
\partial_{t} z(t, x)=F\left(t, x, z(\alpha(t, x)), \partial_{x} z(t, x)\right) .
$$

We require that $\alpha(t, x) \in E$ for $(t, x) \in E$ and $\alpha_{0}(t) \leq t$ for $t \in[0, a]$.

A general class of equations with deviated variables can be obtained in the following way: suppose that $\beta_{0}:[0, a] \rightarrow R, \beta^{\prime}: E \rightarrow R^{n}, \beta^{\prime}=\left(\beta_{1}, \ldots, \beta_{n}\right)$ are given functions and

$$
-h_{0} \leq \beta_{0}(t)-\alpha_{0}(t) \leq 0, \quad-h \leq \beta^{\prime}(t, x)-\alpha^{\prime}(t, x) \leq h, \quad(t, x) \in E .
$$

For the above given function $F$, we define the operator $f$ as follows:

$$
f(t, x, w, q)=F\left(t, x, w\left(\beta_{0}(t)-\alpha_{0}(t), \beta^{\prime}(t, x)-\alpha^{\prime}(t, x)\right), q\right), \quad(t, x, w, q) \in \Omega .(6)
$$

Then

$$
f\left(t, x, z_{\alpha(t, x)}, q\right)=F(t, x, z(\beta(t, x)), q),
$$

where $\beta(t, x)=\left(\beta_{0}(t), \beta^{\prime}(t, x)\right)$ and equation (1) is equivalent to

$$
\partial_{t} z(t, x)=F\left(t, x, z(\beta(t, x)), \partial_{x} z(t, x)\right) .
$$

Now we consider differential integral equations. Suppose that $\gamma_{0}:[0, a] \rightarrow R$, $\gamma^{\prime}: E \rightarrow R^{n}, \gamma^{\prime}=\left(\gamma_{1}, \ldots, \gamma_{n}\right)$ are given functions and

$$
-h_{0} \leq \gamma_{0}(t)-\alpha_{0}(t) \leq 0, \quad-h \leq \gamma^{\prime}(t, x)-\alpha^{\prime}(t, x) \leq h, \quad(t, x) \in E .
$$

For the above given functions $\beta$ and $F$, we define the operator $f$ in the following way:

$$
f(t, x, w, q)=F\left(t, x, \int_{\beta_{0}(t)-\alpha_{0}(t)}^{\gamma_{0}(t)-\alpha_{0}(t)} \int_{\beta^{\prime}(t, x)-\alpha^{\prime}(t, x)}^{\gamma^{\prime}(t, x)-\alpha^{\prime}(t, x)} w(\tau, y) d y d \tau, q\right),
$$

where $(t, x, w, q) \in \Omega$. Then 


$$
f\left(t, x, z_{\alpha(t, x)}, q\right)=F\left(t, x, \int_{\beta(t, x)}^{\gamma(t, x)} z(\tau, y) d y d \tau, q\right)
$$

and (1) reduces to the differential integral equation

$$
\partial_{t} z(t, x)=F\left(t, x, \int_{\beta(t, x)}^{\gamma(t, x)} z(\tau, y) d y d \tau, \partial_{x} z(t, x)\right) .
$$

We will discuss the question of the existence of solutions of problem (1), (2).

Different classes of weak solutions of mixed problems to partial functional differential problems are considered in literature. Almost linear systems in two-independent variables were investigated in $[1,2]$. A continuous function is a solution of a mixed problem considered in the papers if it satisfies an integral functional system arising from functional differential system by integrating along bicharacteristics. Note that papers $[1,2]$ initiated investigations of first order partial functional differential equations.

The class of Carathéodory solutions consists of all functions which are continuous, have their derivatives almost everywhere in a domain and the set of all points where the differential equation or the system is not fulfilled is of Lebesgue measure zero. The existence and uniqueness results for quasilinear systems with initial boundary condition in the class of almost everywhere solutions are given in $[3,4]$. Right-hand sides of equations contain operators of the Volterra type and unknown functions depend on two variables. A general class of mixed problems and Carathéodory solutions for quasilinear equations is investigated in [5]. Functional differential problems considered in these papers are equivalent to integral functional equations which are obtained by integration along bicharacteristics. Under natural assumptions, continuous solutions of functional integral equation are Carathéodory solutions of original problems.

Generalized solutions in the Cinquini Cibrario sense for equations without a functional dependence were first treated in [6-8]. This class of solutions is placed between classical solutions and solutions in the Carathéodory sense. It is important that both inclusions are strict. This class of solutions is investigated in the case that assumptions for given functions are extended. Existence results for mixed problems and nonlinear functional differential equations can be found in [9] and [10] (Chapters IV, V). They are obtained by a quasilinearization procedure and by construction of functional integral systems for unknown functions and for their derivatives with respect to spacial variables. Continuous solutions of integral equations lead to generalized solutions of original problems.

Note that the monograph [11] contains an exposition of generalized solutions in the Cinquini Cibrario sense for nonlinear equations and systems without the functional variable.

Wide classes of solutions to mixed functional differential equations are investigated in $[10,12-14]$. The only derivative with respect to $t$ of unknown functions appear in equations considered in the above papers.

Viscosity solutions of mixed problems for functional differential equations were first treated in $[15,16]$. Uniqueness results were based on a differential inequalities method. Existence theorems were obtained by using the vanishing viscosity method.

Further bibliographical information concerning hyperbolic functional differential equations can be found in the monograph [10].

The paper is a generalization of existence results for nonlinear functional differential equations with initial boundary conditions which are presented in [9] and [10] $($ Chapter V). There are the following differences between the above mentioned results and our theorems.

I. It is assumed in [10] that the function $f$ of the variables $(t, x, w, q)$ has the following property. Write 


$$
\operatorname{sign} \partial_{q} f=\left(\operatorname{sign} \partial_{q_{1}} f, \ldots, \operatorname{sign} \partial_{q_{n}} f\right) .
$$

The following condition is important in [10]: the function $\operatorname{sign} \partial_{q} f$ is constant on $\Omega$. We have omitted the above condition in our considerations. It is assumed in [9] that the function $\operatorname{sign} \partial_{q} f$ is constant on $\Omega$. This condition can be reduced to the assumption adopted in [10] by changing variables in an unknown function in a differential functional equation.

II. The functional dependence in partial differential equations is based on the use of the Hale operator $(t, x) \rightarrow z_{(t, x)}$ where $z_{(t, x)}: D \rightarrow R$. The domain of the function $z_{(t, x)}$ considered in [10] has the form $D=\left[-h_{0}, 0\right] \times\left[0, h^{\prime}\right] \times\left[-h_{0}^{\prime \prime}, 0\right] \subset R^{1+n}$, where $h^{\prime}=$ $=\left(h_{1}, \ldots, h_{k}\right), h^{\prime \prime}=\left(h_{k+1}, \ldots, h_{n}\right)$. In our case we put $D=\left[-h_{0}, 0\right] \times[-h, h]$. It follows that the class of differential equations with deviated variables considered in the paper is more general than an adequate class of equations which can be obtained from [10].

The same conclusion can be drawn for differential integral equations.

III. The right-hand sides of the equations considered in [10] depend on the functional variable $z_{(t, x)}$. In our considerations, equation (1) contains the functional variable $z_{\alpha(t, x)}$ It is easy to see that the class of differential equations which is covered by our theory is more general than a suitable class considered in [10].

The paper is organized as follows. In Section 2 we prove results on the existence and uniqueness and on the regularity of bicharacteristics for nonlinear mixed problems. Integral functional equations generated by (1), (2) are investigated in Section 3. It is shown that under natural assumptions on given functions there exists a sequence of successive approximations and it is covergent. The main results on the existence of generalized solutions and on the continuous dependence of solutions on initial boundary conditions are presented in Section 4. An application to equations with deviated variables is given.

The following function spaces will be needed in our considerations. Write $E_{t}^{*}=$ $=E^{*} \cap\left(\left[-h_{0}, t\right] \times R^{n}\right)$ and $E_{t}=[0, t] \times[-b, b]$ where $0 \leq t \leq a$. We will denote by $\|\cdot\|_{t}$ the supremum norm in the spaces $C\left(E_{t}^{*}, R\right)$ and $C\left(E_{t}^{*}, R^{n}\right)$. Analogously, we will use the symbol $\|\cdot\|_{(t)}$ to denote the supremum norm in $C\left(E_{t}, R\right)$ and $C\left(E_{t}, R^{n}\right)$. We will denote by $M_{n \times n}$ the class of all $n \times n$ matrices with real elements. For $x \in R^{n}, X \in M_{n \times n}$, where $x=\left(x_{1}, \ldots, x_{n}\right)$ and

$$
X=\left[x_{i j}\right]_{i, j=1, \ldots, n}
$$

we put

$$
\|x\|=\sum_{j=1}^{n}\left|x_{i}\right| \quad \text { and } \quad\|X\|=\max _{1 \leq j \leq n} \sum_{i=1}^{n}\left|x_{i j}\right| .
$$

The product of two matrices is denoted by " $\star$ ”. If $X \in M_{n \times n}$ then $X^{T}$ is the transpose matrix. We use the symbol "o" to denote the scalar product in $R^{n}$.

Let us denote by $\|\cdot\|_{0}$ the supremum norm in the space $C(D, R)$. Let $C^{0,1}(D, R)$ be the set of all $w \in C(D, R)$ such that the derivatives $\left(\partial_{x_{1}} w, \ldots, \partial_{x_{n}} w\right)=\partial_{x} w$ exist on $D$ and $\partial_{x} w \in C\left(D, R^{n}\right)$. For $w \in C^{0,1}(D, R)$ we put

$$
\|w\|_{1}=\|w\|_{0}+\max \left\{\left\|\partial_{x} w(t, x)\right\|:(t, x) \in D\right\} .
$$

We denote by $C_{L}^{0,1}(D, R)$ the class of all $w \in C^{0,1}(D, R)$ such that $\|w\|_{1, L}<+\infty$ where 


$$
\|w\|_{1, L}=\|w\|_{1}+\sup \left\{\frac{\left\|\partial_{x} w(t, x)-\partial_{x} w(\bar{t}, \bar{x})\right\|}{|t-\bar{t}|+\|x-\bar{x}\|}:(t, x),(\bar{t}, \bar{x}) \in D,(t, x) \neq(\bar{t}, \bar{x})\right\} .
$$

We will consider the spaces

$$
\Omega=E \times C(D, R) \times R^{n}, \quad \Omega^{(1)}=[-b, b] \times C^{0,1}(D, R) \times R^{n}
$$

and

$$
\Omega^{(1, L)}=[-b, b] \times C_{L}^{0,1}(D, R) \times R^{n} .
$$

Let $\Theta$ be the class of all functions $\gamma \in C\left(R_{+}, R_{+}\right)$which are nondecreasing on $R_{+}$.

Now we define some further function spaces. Given $s=\left(s_{0}, s_{1}, s_{2}\right) \in R_{+}^{3}$, we denote by $C^{1, L}[s]$ the set of all functions $\varphi \in C\left(E_{0} \cup \partial_{0} E, R\right)$ such that

(i) there exists $\partial_{x} \varphi(t, x)$ for $(t, x) \in E_{0} \cup \partial_{0} E$, and

(ii) the estimates $|\varphi(t, x)| \leq s_{0}$,

$$
\begin{gathered}
|\varphi(t, x)-\varphi(\bar{t}, x)| \leq s_{1}|t-\bar{t}|, \quad\left\|\partial_{x} \varphi(t, x)\right\| \leq s_{1}, \\
\left\|\partial_{x} \varphi(t, x)-\partial_{x} \varphi(\bar{t}, \bar{x})\right\| \leq s_{2}[|t-\bar{t}|+\|x-\bar{x}\|],
\end{gathered}
$$

are satisfied on $E_{0} \cup \partial_{0} E$.

Let $\varphi \in C^{1, L}[s]$ be given and let $0<c \leq a, d=\left(d_{0}, d_{1}, d_{2}\right) \in R_{+}^{3}, \quad d_{i} \geq s_{i}$ for $i=0,1,2$, we consider the space $C_{\varphi, c}^{1, L}[d]$ of all functions $z: E_{c}^{*} \rightarrow R$ such that

(i) $z \in C\left(E_{c}^{*}, R\right)$ and $z(t, x)=\varphi(t, x)$ on $\left(E_{0} \cup \partial_{0} E\right) \cap\left(\left[-h_{0}, c\right] \times R^{n}\right)$,

(ii) there exists $\partial_{x} z(t, x)$ on $E_{c}^{*}$ and the estimates $|z(t, x)| \leq d_{0}$, and

$$
\begin{gathered}
|z(t, x)-z(\bar{t}, x)| \leq d_{1}|t-\bar{t}|, \quad\left\|\partial_{x} z(t, x)\right\| \leq d_{1}, \\
\left\|\partial_{x} z(t, x)-\partial_{x} z(\bar{t}, \bar{x})\right\| \leq d_{2}[|t-\bar{t}|+\|x-\bar{x}\|],
\end{gathered}
$$

are satisfied on $E_{c}^{*}$.

Let $p=\left(p_{0}, p_{1}\right) \in R_{+}^{2}, \quad p_{0} \geq s_{1}, \quad p_{1} \geq s_{2}$. We denote by $C_{c}^{0, L}[p]$ the set of all functions $v: E_{c} \rightarrow R^{n}$ such that for $(t, x) \in E_{c}$ the estimates $\|v(t, x)\| \leq p_{0}$ and

$$
\|v(t, x)-v(\bar{t}, \bar{x})\| \leq p_{1}[|t-\bar{t}|+\|x-\bar{x}\|]
$$

hold on $E_{c}$. We will prove that under suitable assumptions on $f, \alpha$ and $\varphi$, and for sufficiently small $c$ with $0<c \leq a$, there exists a solution $\bar{z}$ of the problem (1), (2) such that $\bar{z} \in C_{\varphi, c}^{1, L}[d], \partial_{x} \bar{z} \in C_{c}^{0, L}[p]$. Write

$$
\Delta_{i}^{+}=\left\{x \in[-b, b]: x_{i}=b_{i}\right\}, \quad \Delta_{i}^{-}=\left\{x \in[-b, b]: x_{i}=-b_{i}\right\},
$$

where $1 \leq i \leq n$, and

$$
\Delta=\bigcup_{i=1}^{n}\left(\Delta_{i}^{+} \cup \Delta_{i}^{-}\right)
$$

2. Bicaracteristics of nonlinear equations. We begin with assumptions on $f$.

Assumption $\mathbf{H}\left[\partial_{q} f\right]$. Suppose that the function $f: \Omega \rightarrow R$ of the variables $(t, x$, $w, q), q=\left(q_{1}, \ldots, q_{n}\right)$, is such that

1) $f(\cdot, x, w, q):[0, a] \rightarrow R$ is measurable for each $(x, w, q) \in[-b, b] \times C(D, R) \times$ $\times R^{n}$ and the partial derivatives 


$$
\left(\partial_{q_{1}} f(P), \ldots, \partial_{q_{n}} f(P)\right)=\partial_{q} f(P), \quad P=(t, x, w, q),
$$

exist for $(x, w, q) \in \Omega^{(1)}$ and for almost all $t \in[0, a]$;

2) $\partial_{q} f(\cdot, x, w, q):[0, a] \rightarrow R^{n}$ is measurable and there is $B \in \Theta$ such that

$\left\|\partial_{q} f(t, x, w, q)\right\| \leq B\left(\|w\|_{1}\right) \quad$ for $\quad(x, w, q) \in \Omega^{(1)} \quad$ and for almost all $t \in[0, a]$

and there is $C \in \Theta$ such that for $(x, w, q) \in \Omega^{(1, L)}, \quad(\bar{x}, \bar{q}) \in[-b, b] \times R^{n}$, $\bar{w} \in C^{0,1}(D, R)$ and for almost all $t \in[0, a]$ we have

$$
\left\|\partial_{q} f(t, \bar{x}, w+\bar{w}, \bar{q})-\partial_{q} f(t, x, w, q)\right\| \leq C\left(\|w\|_{1, L}\right)\left[\|x-\bar{x}\|+\|\bar{w}\|_{1}+\|q-\bar{q}\|\right] ;
$$

3) there is $\kappa>0$ such that for $1 \leq i \leq n$ we have

$$
\partial_{q_{i}} f(t, x, w, q) \geq \kappa \text { for }(x, w, q) \in \Delta_{i}^{+} \times C^{0,1}(D, R) \times R^{n}
$$

and

$$
\partial_{q_{i}} f(t, x, w, q) \leq-\kappa \quad \text { for } \quad(x, w, q) \in \Delta_{i}^{-} \times C^{0,1}(D, R) \times R^{n}
$$

for almost all $t \in[0, a]$.

Assumption $\mathbf{H}[\boldsymbol{\alpha}]$. Suppose that the functions $\alpha:[0, a] \rightarrow[0, a]$ and $\alpha^{\prime}: E \rightarrow$ $\rightarrow[-b, b]$ are such that

1) $\alpha_{0}(t) \leq t$ for $t \in[0, a]$ and there is $r_{0} \in R_{+}$such that

$$
\left|\alpha_{0}(t)-\alpha_{0}(\bar{t})\right| \leq r_{0}|t-\bar{t}| \quad \text { on } \quad[0, a] ;
$$

2) $\alpha^{\prime}$ is of class $C^{1}$ and

$$
\left\|\partial_{x} \alpha^{\prime}(t, x)\right\| \leq r_{0} \quad \text { on } \quad E
$$

3) there is $r_{1} \in R_{+}$such that

$$
\left\|\partial_{x} \alpha^{\prime}(t, x)-\partial_{x} \alpha^{\prime}(\bar{t}, \bar{x})\right\| \leq r_{1}\|x-\bar{x}\| \quad \text { on } \quad E .
$$

Write $r=\left(r_{0}, r_{1}\right)$.

Suppose that $\varphi \in C^{1, L}[s]$ and $z \in C_{\varphi, c}^{1, L}[d], \quad u \in C_{c}^{0, L}[p]$. We consider the Cauchy problem

$$
\eta^{\prime}(\tau)=-\partial_{q} f\left(\tau, \eta(\tau), z_{\alpha(\tau, \eta(\tau))}, u(\tau, \eta(\tau))\right), \quad \eta(t)=x,
$$

and denote by $g[z, u](\cdot, t, x)$ its solution in the Carathéodory sense. The function $g[z, u](\cdot, t, x)$ is the bicharacteristic of equation (1) corresponding to $(z, u)$. Let $I_{(t, x)}$ be the domain of $g[z, u](\cdot, t, x)$ and $\delta[z, u](t, x)$ is the left end of the maximal interval on which the bicharacteristic $g[z, u](\cdot, t, x)$ is defined. Write

$$
P[z, u](\tau, t, x)=\left(\tau, g[z, u](\tau, t, x), z_{\alpha(\tau, g[z, u](\tau, t, x))}, u(\tau, g[z, u](\tau, t, x))\right) .
$$

We prove a lemma on bicharacteristics.

Lemma 1. Suppose that Assumptions $\mathrm{H}\left[\partial_{q} f\right], \mathrm{H}[\alpha]$ are satisfied and let

$$
\varphi, \bar{\varphi} \in C^{1, L}[s], \quad z \in C_{\varphi, c}^{1, L}[d], \quad \bar{z} \in C_{\bar{\varphi}, c}^{1, L}[d], \quad u, \bar{u} \in C_{c}^{0, L}[p],
$$

be given. Then the solutions $g[z, u](\cdot, t, x)$ and $g[\bar{z}, \bar{u}](\cdot, t, x)$ exist on the intervals $I_{(t, x)}$ and $\bar{I}_{(t, x)}$ such that for $\xi=\delta[z, u](t, x), \quad \bar{\xi}=\delta[\bar{z}, \bar{u}](t, x)$ we have $g[z, u](\xi, t, x) \in \Delta$ and $g[\bar{z}, \bar{u}](\bar{\xi}, t, x) \in \Delta$. The bicharacteristics are unique on $I_{(t, x)}$ and $\bar{I}_{(t, x)}$. Moreover we have the estimates 
$\|g[z, u](\tau, t, x)-g[z, u](\tau, \bar{t}, \bar{x})\| \leq \bar{C}[|t-\bar{t}|+\|x-\bar{x}\|]$
for $\tau \in I_{(t, x)} \cap I_{(\bar{t}, \bar{x})}, \quad(t, x),(\bar{t}, \bar{x}) \in E_{c}$, and
$\|g[z, u](\tau, t, x)-g[\bar{z}, \bar{u}](\tau, t, x)\| \leq \bar{C}\left|\int_{t}^{\tau}\left[\|z-\bar{z}\|_{\xi}+\left\|\partial_{x} z-\partial_{x} \bar{z}\right\|_{(\xi)}+\|u-\bar{u}\|_{(\xi)}\right] d \xi\right|$

for $\tau \in I_{(t, x)} \cap \bar{I}_{(t, x)}, \quad(t, x) \in E_{c}$, where $\bar{I}_{(t, x)}$ is the domain of the bicharacteristic $g[\bar{z}, \bar{u}](\cdot, t, x)$ and

$$
\bar{C}=\max \{1, B(\tilde{d}), C(|d|)\} \exp \left\{c C(|d|)\left[1+r_{0}\left(d_{1}+d_{2}\right)+p_{1}\right]\right\}
$$

and

$$
\tilde{d}=d_{0}+d_{1}, \quad|d|=d_{0}+d_{1}+d_{2} .
$$

Proof. The existence and uniqueness of solutions of (11) follows from the classical theorem on Carathéodory solutions of initial problems. The function $g[z, u](\cdot, t, x)$ satisfies the integral equation

$$
g[z, u](\tau, t, x)=x-\int_{t}^{\tau} \partial_{q} f(P[z, u](\xi, t, x)) d \xi .
$$

Since $z \in C_{\varphi, c}^{1, L}[d]$ condition 2 of Assumption $\mathrm{H}[\alpha]$ shows that

$$
\left\|z_{\alpha(\tau, y)}-z_{\alpha(\tau, \bar{y})}\right\|_{1} \leq r_{0}\left(d_{1}+d_{2}\right)\|y-\bar{y}\|
$$

for all $(\tau, y),(\tau, \bar{y}) \in E_{c}$. It follows from Assumption $\mathrm{H}\left[\partial_{q} f\right]$ that the function $g[z, u](\cdot, t, x)-g[z, u](\cdot, \bar{t}, \bar{x})$ satisfies the integral inequality

$$
\begin{gathered}
\|g[z, u](\tau, t, x)-g[z, u](\tau, \bar{t}, \bar{x})\| \leq \max \{1, B(\tilde{d})\}[|t-\bar{t}|+\|x-\bar{x}\|]+ \\
+C(|d|)\left[1+r_{0}\left(d_{1}+d_{2}\right)+p_{1}\right]\left|\int_{t}^{\tau}\|g[z, u](\xi, t, x)-g[z, u](\xi, \bar{t}, \bar{x})\| d \xi\right|, \\
\tau \in I_{(t, x)} \cap I_{(\bar{t}, \bar{x})} .
\end{gathered}
$$

Now we obtain (12) by the Gronwall inequality. For $(\tau, y),(\tau, \bar{y}) \in E_{c}$ we have the estimate

$$
\left\|z_{\alpha(\tau, y)}-\bar{z}_{\alpha(\tau, \bar{y})}\right\|_{1} \leq\|z-\bar{z}\|_{\tau}+\left\|\partial_{x} z-\partial_{x} \bar{z}\right\|_{(\tau)}+r_{0}\left(d_{1}+d_{2}\right)\|y-\bar{y}\| .
$$

It follows that the function $g[z, u](\cdot, t, x)-g[\bar{z}, \bar{u}](\cdot, t, x)$ satisfies the integral inequality

$$
\begin{gathered}
\|g[z, u](\tau, t, x)-g[\bar{z}, \bar{u}](\tau, t, x)\| \leq \\
\leq C(|d|)\left|\int_{t}^{\tau}\left[\|z-\bar{z}\|_{\xi}+\left\|\partial_{x} z-\partial_{x} \bar{z}\right\|_{(\xi)}+\|u-\bar{u}\|_{(\xi)}\right] d \xi\right|+ \\
+C(|d|)\left[1+r_{0}\left(d_{1}+d_{2}\right)+p_{1}\right]\left|\int_{t}^{\tau}\|g[z, u](\xi, t, x)-g[\bar{z}, \bar{u}](\xi, t, x)\| d \xi\right|, \\
\tau \in I_{(t, x)} \cap \bar{I}_{(t, x)} .
\end{gathered}
$$

ISSN 1027-3190. Укр. мат. журн., 2006, m. 58, № 6 
We get (13) from the Gronwall inequality. This proves Lemma 1.

Now we give a lemma on a regularity of the function $\delta[z, u]$.

Lemma 2. Suppose that Assumptions $\mathrm{H}\left[\partial_{q} f\right], \mathrm{H}[\alpha]$ are satisfied and

$$
\varphi, \bar{\varphi} \in C^{1, L}[s], \quad z \in C_{\varphi, c}^{1, L}[d], \quad \bar{z} \in C_{\bar{\varphi}, c}^{1, L}[d], \quad u, \bar{u} \in C_{c}^{0, L}[p] .
$$

Then the functions $\delta[z, u]$ and $\delta[\bar{z}, \bar{u}]$ are continuous on $E_{c}$. Moreover we have the estimates

$$
|\delta[z, u](t, x)-\delta[z, u](\bar{t}, \bar{x})| \leq \frac{2 \bar{C}}{\kappa}[|t-\bar{t}|+\|x-\bar{x}\|]
$$

where $(t, x),(\bar{t}, \bar{x}) \in E_{c}$ and

$$
\begin{aligned}
& \qquad|\delta[z, u](t, x)-\delta[\bar{z}, \bar{u}](t, x)| \leq \frac{2 \bar{C}}{\kappa} \int_{0}^{t}\left[\|z-\bar{z}\|_{\xi}+\left\|\partial_{x} z-\partial_{x} \bar{z}\right\|_{(\xi)}+\|u-\bar{u}\|_{(\xi)}\right] d \xi \\
& \text { where }(t, x) \in E_{c} .
\end{aligned}
$$

Proof. The continuity of $\delta[z, u]$ and $\delta[\bar{z}, \bar{u}]$ on $E_{c}$ follows from classical theorems on continuous dependence on initial conditions for Carathéodory solutions of initial problems. Now we prove (15). This estimate is obvious in the case $\delta[z, u](t, x)=$ $=\delta[z, u](\bar{t}, \bar{x})=0$ (i.e., in the case when solutions of problem (11) are defined on $[0, t]$ and $[0, \bar{t}])$. Suppose now that $0 \leq \delta[z, u](t, x)<\delta[z, u](\bar{t}, \bar{x})$. Then for $\bar{\zeta}=\delta[z, u](\bar{t}, \bar{x})$ we have $g[z, u](\bar{\zeta}, \bar{t}, \bar{x}) \in \Delta$ and there exists $i, 1 \leq i \leq n$, such that $\left|g_{i}[z, u](\bar{\zeta}, \bar{t}, \bar{x})\right|=b_{i}$. Two possibilities can occur, either (i) $g_{i}[z, u](\bar{\zeta}, \bar{t}, \bar{x})=b_{i}$ or (ii) $g_{i}[z, u](\bar{\zeta}, \bar{t}, \bar{x})=-b_{i}$. Consider the case (i). Let $x=\left(x_{1}, \ldots, x_{n}\right), \tilde{x}=$ $=\left(x_{1}, \ldots, x_{i-1}, b_{i}, x_{i+1}, \ldots, x_{n}\right)$. We have the estimate

$$
\left|\partial_{q_{i}} f\left(t, x, z_{\alpha(t, x)}, u(t, x)\right)-\partial_{q_{i}} f\left(t, \tilde{x}, z_{\alpha(t, \tilde{x})}, u(t, \tilde{x})\right)\right| \leq \tilde{c}\left(b_{i}-x_{i}\right),
$$

for $(t, x) \in E_{c}$, where $\tilde{c}=C(|d|)\left[1+r_{0}\left(d_{1}+d_{2}\right)+p_{1}\right]$. Thus

$$
\partial_{q_{i}} f\left(t, x, z_{\alpha(t, x)}, u(t, x)\right) \geq \frac{\kappa}{2}
$$

for $(t, x) \in E_{c}$ such that $b_{i}-x_{i} \leq \kappa(2 \tilde{c})^{-1}$. It follows from Lemma 1, that

$$
b_{i}-g_{i}[z, u](\bar{\zeta}, t, x)=g_{i}[z, u](\bar{\zeta}, \bar{t}, \bar{x})-g_{i}[z, u](\bar{\zeta}, t, x) \leq \frac{\kappa}{2 \tilde{c}},
$$

for $(t, x),(\bar{t}, \bar{x}) \in E_{c}$ such that

$$
|t-\bar{t}|+\|x-\bar{x}\| \leq \frac{\kappa}{2 \tilde{c} \bar{C}}
$$

Then we get

$$
\partial_{q_{i}} f\left(\bar{\zeta}, g[z, u](\bar{\zeta}, t, x), z_{\alpha(\bar{\zeta}, g[z, u](\bar{\zeta}, t, x))}, u(\bar{\zeta}, g[z, u](\bar{\zeta}, t, x))\right) \geq \frac{\kappa}{2}>0
$$

and consequently,

$$
\partial_{t} g_{i}[z, u](\delta[z, u](\bar{t}, \bar{x}), t, x)<0
$$

for $(t, x),(\bar{t}, \bar{x}) \in E_{c}$ satisfying (18). It can be easily seen that $g_{i}[z, u](\cdot, t, x)$ is decreasing on the interval $(\delta[z, u](t, x), \delta[z, u](\bar{t}, \bar{x}))$. Therefore 


$$
b_{i}-g_{i}[z, u](\tau, t, x) \leq \frac{\kappa}{2 \tilde{c}}
$$

and the estimate

$$
\partial_{q_{i}} f\left(\tau, g[z, u](\tau, t, x), z_{\alpha(\tau, g[z, u](\tau, t, x))}, u(\tau, g[z, u](\tau, t, x))\right) \geq \frac{\kappa}{2}
$$

holds for $\tau \in(\delta[z, u](t, x), \delta[z, u](\bar{t}, \bar{x}))$ and $(t, x),(\bar{t}, \bar{x}) \in E_{c}$ such that (18) is satisfied. Then

$$
\begin{gathered}
-\frac{\kappa}{2}[\delta[z, u](\bar{t}, \bar{x})-\delta[z, u](t, x)] \geq \\
\geq-\int_{\delta[z, u](t, x)}^{\delta[z, u](\bar{t}, \bar{x})} \partial_{q_{i}} f\left(\tau, g[z, u](\tau, t, x), z_{\alpha(\tau, g[z, u](\tau, t, x))}, u(\tau, g[z, u](\tau, t, x))\right) d \tau= \\
=g_{i}[z, u](\delta[z, u](\bar{t}, \bar{x}), t, x)-g_{i}[z, u](\delta[z, u](t, x), t, x) \geq \\
\geq g_{i}[z, u](\delta[z, u](\bar{t}, \bar{x}), t, x)-g_{i}[z, u](\delta[z, u](\bar{t}, \bar{x}), \bar{t}, \bar{x}) \geq \\
\geq-\bar{C}[|t-\bar{t}|+\|x-\bar{x}\|] .
\end{gathered}
$$

Thus the proof of (15) for $(t, x),(\bar{t}, \bar{x}) \in E_{c}$, such that (18) holds, is completed in the case (i). In a similar way we prove (ii). Let $(t, x),(\bar{t}, \bar{x}) \in E_{c}$ be arbitrary. We put $M=\|x-\bar{x}\|+|t-\bar{t}|$. There exists $K \in \mathbf{N}$ such that

$$
(K-1) \frac{\kappa}{2 \tilde{c} \bar{C}}<M \leq K \frac{\kappa}{2 \tilde{c} \bar{C}} .
$$

Let $\varepsilon \in \mathbf{R}, \varepsilon=1 / K$. For $j=0, \ldots, K$ we put

$$
\bar{x}^{(j)}=j \varepsilon \bar{x}+(1-j \varepsilon) x, \quad \bar{t}^{(j)}=j \varepsilon \bar{t}+(1-j \varepsilon) t .
$$

Note that $\left(\bar{t}^{(0)}, \bar{x}^{(0)}\right)=(t, x),\left(\bar{t}^{(K)}, \bar{x}^{(K)}\right)=(\bar{t}, \bar{x})$ and

$$
\left\|\bar{x}^{(j)}-\bar{x}^{(j+1)}\right\|+\left|\bar{t}^{(j)}-\bar{t}^{(j+1)}\right|=\frac{M}{K} \leq \frac{\kappa}{2 \tilde{c} \bar{C}}
$$

for $j=0, \ldots, K-1$. It is easy to see that

$$
\|x-\bar{x}\|=\sum_{j=0}^{K-1}\left\|\bar{x}^{(j)}-\bar{x}^{(j+1)}\right\| \quad \text { and } \quad\|t-\bar{t}\|=\sum_{j=0}^{K-1}\left\|\bar{t}^{(j)}-\bar{t}^{(j+1)}\right\| .
$$

Then we have

$$
\begin{gathered}
|\delta[z, u](t, x)-\delta[z, u](\bar{t}, \bar{x})| \leq \\
\leq \sum_{j=0}^{K-1}\left|\delta[z, u]\left(\bar{t}^{(j)}, \bar{x}^{(j)}\right)-\delta[z, u]\left(\bar{t}^{(j+1)}, \bar{x}^{(j+1)}\right)\right| \leq \\
\leq \sum_{j=0}^{K-1} \frac{2 \bar{C}}{\kappa}\left[\left|\bar{t}^{(j)}-\bar{t}^{(j+1)}\right|+\left\|\bar{x}^{(j)}-\bar{x}^{(j+1)}\right\|\right]=\frac{2 \bar{C}}{\kappa}[|t-\bar{t}|+\|x-\bar{x}\|] .
\end{gathered}
$$

Thus we see that (15) holds true for all $(t, x),(\bar{t}, \bar{x}) \in E_{c}$. Now we consider estimate (16). The inequality is obvious if $\delta[z, u](t, x)=\delta[\bar{z}, \bar{u}](t, x)=0$. Suppose now that $0 \leq \delta[z, u](t, x)<\delta[\bar{z}, \bar{u}](t, x)$. Then for $\zeta=\delta[\bar{z}, \bar{u}](t, x)$ we have $\delta[\bar{z}, \bar{u}](\zeta, t, x) \in \Delta$ and there is $i, 1 \leq i \leq n$, such that $\left|g_{i}[\bar{z}, \bar{u}](\zeta, t, x)\right|=b_{i}$. Two possibilities can happen, either (i) $g_{i}[\bar{z}, \bar{u}](\zeta, t, x)=b_{i}$ or (ii) $g_{i}[\bar{z}, \bar{u}](\zeta, t, x)=-b_{i}$. 
Consider the case (i). We have the estimate (17) for $(t, x) \in E_{c}$.

It follows from Lemma 1, that

$$
\left|g_{i}[z, u](\zeta, t, x)-g_{i}[\bar{z}, \bar{u}](\zeta, t, x)\right| \leq c \bar{C}\left[\|z-\bar{z}\|_{t}+\left\|\partial_{x} z-\partial_{x} \bar{z}\right\|_{(t)}+\|u-\bar{u}\|_{(t)}\right] .
$$

Thus we have

$$
b_{i}-g_{i}[z, u](\zeta, t, x)=g_{i}[\bar{z}, \bar{u}](\zeta, t, x)-g_{i}[z, u](\zeta, t, x) \leq \frac{\kappa}{2 \tilde{c}},
$$

for $(t, x) \in E_{c}$ and $z, \bar{z}, u, \bar{u}$ such that

$$
\|z-\bar{z}\|_{t}+\left\|\partial_{x} z-\partial_{x} \bar{z}\right\|_{(t)}+\|u-\bar{u}\|_{(t)} \leq \frac{\kappa}{2 c \tilde{c} \bar{C}} .
$$

Then we get

$$
\partial_{q_{i}} f\left(\zeta, g[z, u](\zeta, t, x), z_{\alpha(\zeta, g[z, u](\zeta, t, x))}, u(\zeta, g[z, u](\zeta, t, x))\right) \geq \frac{\kappa}{2}>0,
$$

and consequently,

$$
\partial_{t} g_{i}[z, u](\delta[\bar{z}, \bar{u}](t, x), t, x)<0
$$

for $(t, x) \in E_{c}$ and for $z, \bar{z}, u, \bar{u}$ satisfying (19). It can be easily seen that $g_{i}[z, u](\cdot, t, x)$ is decreasing on $(\delta[z, u](t, x), \delta[\bar{z}, \bar{u}](t, x))$. Therefore

$$
b_{i}-g_{i}[z, u](\tau, t, x) \leq \frac{\kappa}{2 \tilde{c}}
$$

and the estimate

$$
\partial_{q_{i}} f\left(\tau, g[z, u](\tau, t, x), z_{\alpha(\tau, g[z, u](\tau, t, x))}, u(\tau, g[z, u](\tau, t, x))\right) \geq \frac{\kappa}{2}
$$

holds for $\tau \in(\delta[z, u](t, x), \delta[\bar{z}, \bar{u}](t, x)),(t, x) \in E_{c}$ and $z, \bar{z}, u, \bar{u}$ such that (19) is satisfied. Then

$$
-\frac{\kappa}{2}[\delta[\bar{z}, \bar{u}](t, x)-\delta[z, u](t, x)] \geq
$$

$$
\begin{gathered}
\geq-\int_{\delta[z, u](t, x)}^{\delta[\bar{z}, \bar{u}](t, x)} \partial_{q_{i}} f\left(\tau, g[z, u](\tau, t, x), z_{\alpha(\tau, g[z, u](\tau, t, x))}, u(\tau, g[z, u](\tau, t, x))\right) d \tau \geq \\
\geq g_{i}[z, u](\delta[\bar{z}, \bar{u}](t, x), t, x)-g_{i}[\bar{z}, \bar{u}](\delta[\bar{z}, \bar{u}](t, x), t, x) \geq \\
\geq-\bar{C}\left|\int_{t}^{\delta[\bar{z}, \bar{u}](t, x)}\left[\|z-\bar{z}\|_{\xi}+\left\|\partial_{x} z-\partial_{x} \bar{z}\right\|_{(\xi)}+\|u-\bar{u}\|_{(\xi)}\right] d \xi\right| \geq \\
\geq-\bar{C} \int_{0}^{t}\left[\|z-\bar{z}\|_{\xi}+\left\|\partial_{x} z-\partial_{x} \bar{z}\right\|_{(\xi)}+\|u-\bar{u}\|_{(\xi)}\right] d \xi .
\end{gathered}
$$

Thus the proof of (16) for $(t, x) \in E_{c}$ and for $z, \bar{z}, u, \bar{u}$ such that (19) holds, is completed in the case (i). In a similar way we prove (ii).

Let $z \in C_{\varphi, c}^{1, L}[d], \bar{z} \in C_{\bar{\varphi}, c}^{1, L}[d], u, \bar{u} \in C^{0, L}[p]$ be arbitrary. We put $M=\|z-\bar{z}\|_{t}+$ $+\left\|\partial_{x} z-\partial_{x} \bar{z}\right\|_{(t)}+\|u-\bar{u}\|_{(t)}$. There exists $K \in \mathbf{N}$ such that

$$
(K-1) \frac{\kappa}{2 c \tilde{c} \bar{C}}<M \leq K \frac{\kappa}{2 c \tilde{c} \bar{C}} .
$$

ISSN 1027-3190. Укр. мат. журн., 2006, m. 58, № 6 
Let $\varepsilon \in \mathbf{R}, \varepsilon=1 / K$. For $j=0, \ldots, K$ we put

$$
\bar{z}_{j}=j \varepsilon \bar{z}+(1-j \varepsilon) z \text { and } \quad \bar{u}_{j}=j \varepsilon \bar{u}+(1-j \varepsilon) u \text { on } E_{c} .
$$

Note that $\bar{z}_{0}=z, \bar{u}_{0}=u, \bar{z}_{K}=\bar{z}, \bar{u}_{K}=\bar{u}$ and

$$
\left\|\bar{z}_{j}-\bar{z}_{j+1}\right\|_{t}+\left\|\partial_{x} \bar{z}_{j}-\partial_{x} \bar{z}_{j+1}\right\|_{(t)}+\left\|\bar{u}_{j}-\bar{u}_{j+1}\right\|_{(t)}=\frac{M}{K} \leq \frac{\kappa}{2 c \tilde{c} \bar{C}}
$$

for $j=0, \ldots, K-1$. It is easy to see that

$$
\|z-\bar{z}\|_{t}=\sum_{j=0}^{K-1}\left\|\bar{z}_{j}-\bar{z}_{j+1}\right\|_{t}, \quad\left\|\partial_{x} z-\partial_{x} \bar{z}\right\|_{(t)}=\sum_{j=0}^{K-1}\left\|\partial_{x} \bar{z}_{j}-\partial_{x} \bar{z}_{j+1}\right\|_{(t)}
$$

and

$$
\|u-\bar{u}\|_{(t)}=\sum_{j=0}^{K-1}\left\|\bar{u}_{j}-\bar{u}_{j+1}\right\|_{(t)}
$$

Then we have

$$
\begin{gathered}
|\delta[z, u](t, x)-\delta[\bar{z}, \bar{u}](t, x)| \leq \\
\leq \sum_{j=0}^{K-1}\left|\delta\left[\bar{z}_{j}, \bar{u}_{j}\right](t, x)-\delta\left[\bar{z}_{j+1}, \bar{u}_{j+1}\right](t, x)\right| \leq \\
\leq \sum_{j=0}^{K-1} \frac{2 \bar{C}}{\kappa} \int_{0}^{t}\left[\left\|\bar{z}_{j}-\bar{z}_{j+1}\right\|_{\xi}+\left\|\partial_{x} \bar{z}_{j}-\partial_{x} \bar{z}_{j+1}\right\|_{(\xi)}+\left\|\bar{u}_{j}-\bar{u}_{j+1}\right\|_{(\xi)}\right] d \xi= \\
=\frac{2 \bar{C}}{\kappa} \int_{0}^{t}\left[\|z-\bar{z}\|_{\xi}+\left\|\partial_{x} z-\partial_{x} \bar{z}\right\|_{(\xi)}+\|u-\bar{u}\|_{(\xi)}\right] d \xi .
\end{gathered}
$$

Therefore (16) holds true for all $z \in C_{\varphi, c}^{1, L}[d], \quad \bar{z} \in C_{\bar{\varphi}, c}^{1, L}[d], \quad u, \quad \bar{u} \in C_{c}^{0, L}[p] . \quad$ This completes the proof of the Lemma 2.

3. Integral functional equations. We denote by $C L(D, R)$ the set of all continuous and real functions defined on $C(D, R)$ and by $\|\cdot\|_{\star}$ the norm in $C L\left(D, R^{n}\right)$. We formulate now next assumptions on $f$.

Assumption $\mathbf{H}[\boldsymbol{f}]$. Suppose that the Assumption $\mathrm{H}\left[\partial_{q} f\right]$ is satisfied and

1) there is $B_{0} \in \Theta$ such that

$$
|f(t, x, w, q)| \leq B_{0}\left(\|w\|_{0}\right) \quad \text { on } \quad \Omega
$$

2) the partial derivatives

$$
\left(\partial_{x_{1}} f(P), \ldots, \partial_{x_{n}} f(P)\right)=\partial_{x} f(P), \quad P=(t, x, w, q),
$$

and the Fréchet derivative $\partial_{w} f(P)$ exist for $(x, w, q) \in \Omega$ and for almost all $t \in$ $\in[0, a]$

3) the estimates

$$
\left\|\partial_{x} f(t, x, w, q)\right\| \leq B\left(\|w\|_{1}\right), \quad\left\|\partial_{w} f(t, x, w, q)\right\|_{\star} \leq B\left(\|w\|_{1}\right),
$$

are satisfied for $(x, w, q) \in \Omega^{(1)}$ and for almost all $t \in[0, a]$;

4) the terms

$$
\left\|\partial_{x} f(t, \bar{x}, \bar{w}+w, \bar{q})-\partial_{x} f(t, x, w, q)\right\|,
$$




$$
\left\|\partial_{w} f(t, \bar{x}, \bar{w}+w, \bar{q})-\partial_{w} f(t, x, w, q)\right\|_{\star}
$$

are bounded from above by

$$
C\left(\|w\|_{1, L}\right)\left[\|x-\bar{x}\|+\|\bar{w}\|_{1}+\|q-\bar{q}\|\right]
$$

for $(x, w, q) \in \Omega^{(1, L)}, \quad(\bar{x}, \bar{q}) \in[-b, b] \times R^{n}, \quad \bar{w} \in C^{0,1}(D, R) \quad$ and for almost all $t \in$ $\in[0, a]$.

Remark 1. We give a theorem on the existence of solutions of the problem (1), (2). For simplicity of formulation of the result we have assumed the same estimates for the derivatives $\partial_{x} f, \partial_{w} f, \partial_{q} f$. We also have assumed the Lipschitz condition for these derivatives with the same coefficient.

Suppose that $\varphi \in C^{1, L}[s]$. Let $C_{\varphi, c}^{0, L}[p]$ be the class of all functions $u: E_{c}^{*} \rightarrow R^{n}$ such that $u(t, x)=\partial_{x} \varphi(t, x)$ on $\left(E_{0} \cup \partial_{0} E\right) \cap\left(\left[-h_{0}, c\right] \times R^{n}\right)$ and $\left.u\right|_{E_{c}} \in C_{c}^{0, L}[p]$. Now we formulate a system of inegral equations which are generated by (1), (2). Write

$$
\begin{gathered}
Q[z, u](t, x)=(\delta[z, u](t, x), g[z, u](\delta[z, u](t, x), t, x)), \\
\Phi[z, u](t, x)=\varphi(Q[z, u](t, x)), \\
\psi[z, u](t, x)=\partial_{x} \varphi(Q[z, u](t, x)), \quad \psi=\left(\psi_{1}, \ldots, \psi_{n}\right), \\
W[z, u](\tau, t, x)=u_{\alpha(\tau, g[z, u](\tau, t, x))} \star \partial_{x} \alpha(\tau, g[z, u](\tau, t, x)) .
\end{gathered}
$$

Given $\varphi \in C^{1, L}[s], \quad z \in C_{\varphi, c}^{1, L}[d]$, and $u \in C_{\varphi, c}^{0, L}[p]$, where $0<c \leq a$. We define

$$
\begin{gathered}
F[z, u](t, x)=\Phi[z, u](t, x)+ \\
+\int_{\delta[z, u](t, x)}^{t}\left[f(P[z, u](\tau, t, x))-\partial_{q} f(P[z, u](\tau, t, x)) \circ u(\tau, g[z, u](\tau, t, x))\right] d \tau,
\end{gathered}
$$

and

$$
\begin{gathered}
G[z, u](t, x)=\psi[z, u](t, x)+\int_{\delta[z, u](t, x)}^{t}\left[\partial_{x} f(P[z, u](\tau, t, x))+\right. \\
\left.+\partial_{w} f(P[z, u](\tau, t, x)) W[z, u](\tau, t, x)\right] d \tau .
\end{gathered}
$$

We will consider the following system of functional integral equations:

$$
\begin{gathered}
z(t, x)=F[z, u](t, x), \quad u(t, x)=G[z, u](t, x), \\
g[z, u](\tau, t, x)=x+\int_{\tau}^{t} \partial_{q} f(P[z, u](\xi, t, x)) d \xi
\end{gathered}
$$

with initial-boundary conditions

$$
z=\varphi, \quad u=\partial_{x} \varphi \quad \text { on } \quad\left(E_{0} \cup \partial_{0} E\right) \cap\left(\left[-h_{0}, c\right] \times R^{n}\right) .
$$

Remark 2. Integral functional system (20) - (22) is obtained in the following way. We introduce first an additional unknown function $u$, where $u=\partial_{x} z$. Then we consider the linearization of (1) with respect to $u$, i.e.,

$$
\partial_{t} z(t, x)=f(U)+\partial_{q} f(U) \circ\left(\partial_{x} z(t, x)-u(t, x)\right),
$$

where $U=\left(t, x, z_{\alpha(t, x)}, u(t, x)\right)$. By virtue of equation (1) we get the differential system for the unknown function $u$ : 


$$
\begin{aligned}
\partial_{t} u(t, x) & =\partial_{x} f(U)+\partial_{q} f(U) \star\left[\partial_{x} u(t, x)\right]^{T}+ \\
+ & \partial_{w} f(U)\left(\partial_{x} z\right)_{\alpha(t, x)} \star \partial_{x} \alpha(t, x) .
\end{aligned}
$$

Finally we put $\partial_{x} z=u$ in (24). System (23), (24) has the following property: the differential equations of bicharacteristics for (23) and for (24) are the same and they have the form (11).

If we consider (23), (24) along the bicharacteristics $g[z, u](\cdot, t, x)$, we obtain

$\frac{d}{d \tau} z(\tau, g[z, u](\tau, t, x))=f(P[z, u](\tau, t, x))-\partial_{q} f(P[z, u](\tau, t, x)) \circ u(\tau, g[z, u](\tau, t, x))$, and

$$
\frac{d}{d \tau} u(\tau, g[z, u](\tau, t, x))=\partial_{x} f(P[z, u](\tau, t, x))+\partial_{w} f(P[z, u](\tau, t, x)) W[z, u](\tau, t, x) .
$$

By integrating the above equations on $[\delta[z, u](t, x), t]$ with respect to $\tau$, we get (20), (21).

The existence results for (20) - (22) is based on the following method of the successive approximations. Suppose that $\varphi \in C^{1, L}[s]$ and that Assumption $H[f]$ are satisfied. We define the sequence

$$
\left\{z^{(m)}, u^{(m)}\right\}, \quad z^{(m)}: E_{c}^{*} \rightarrow R, \quad u^{(m)}: E_{c}^{*} \rightarrow R^{n},
$$

in the following way. Let $z^{(0)}: E_{c}^{*} \rightarrow R, u^{(0)}: E_{c}^{*} \rightarrow R^{n}$ be arbitrary functions such that $z^{(0)} \in C_{\varphi, c}^{1, L}[d], \quad u^{(0)} \in C_{\varphi, c}^{0, L}[p]$ and $\partial_{x} z^{(0)}(t, x)=u^{(0)}(t, x)$ on $E_{c}$. Now, if $\left(z^{(m)}, u^{(m)}\right) \in C_{\varphi, c}^{1, L}[d] \times C_{\varphi, c}^{0, L}[p]$ are known functions, then $u^{(m+1)}$ is a solution of the equation

$$
u=G^{(m)}[u]
$$

with the initial-boundary condition

$$
u(t, x)=\partial_{x} \varphi(t, x) \quad \text { on } \quad\left(E_{0} \cup \partial_{0} E\right) \cap\left(\left[-h_{0}, c\right] \times R^{n}\right)
$$

and

$$
\begin{gathered}
z^{(m+1)}(t, x)=F\left[z^{(m)}, u^{(m+1)}\right](t, x) \quad \text { on } \quad E_{c}, \\
z^{(m+1)}(t, x)=\varphi(t, x) \quad \text { on } \quad\left(E_{0} \cup \partial_{0} E\right) \cap\left(\left[-h_{0}, c\right] \times R^{n}\right),
\end{gathered}
$$

where $G^{(m)}=\left(G_{1}^{(m)}, \ldots, G_{n}^{(m)}\right)$ is defined by

$$
\begin{gathered}
G^{(m)}[u](t, x)=\psi\left[z^{(m)}, u\right](t, x)+\int_{\delta\left[z^{(m)}, u\right](t, x)}^{t}\left[\partial_{x} f\left(P\left[z^{(m)}, u\right](\tau, t, x)\right)+\right. \\
\left.+\partial_{w} f\left(P\left[z^{(m)}, u\right](\tau, t, x)\right) W^{(m)}\left[z^{(m)}, u\right](\tau, t, x)\right] d \tau,
\end{gathered}
$$

and

$$
W^{(m)}[z, u](\tau, t, x)=\left(u^{(m)}\right)_{\alpha(\tau, g[z, u](\tau, t, x))} \star \partial_{x} \alpha(\tau, g[z, u](\tau, t, x)) .
$$

We wish to emphasize that the main difficulty in carrying out this construction is the problem of the existence of the sequence $\left\{z^{(m)}, u^{(m)}\right\}$.

Put

$$
\tilde{r}=1+r_{0}\left(d_{1}+d_{2}\right)+p_{1},
$$

ISSN 1027-3190. Укр. мат. журн., 2006, m. 58, № 6 


$$
\begin{gathered}
A_{0}=\bar{C}\left(1+p_{0} r_{0}\right)\left[\frac{2 B(\tilde{d})}{\kappa}+\tilde{r} c C(|d|)\right]+\bar{C} c B(\tilde{d})\left(p_{1} r_{0}^{2}+p_{0} r_{1}\right), \\
\bar{A}=A_{0}+s_{2} \bar{C}\left[1+\frac{2}{\kappa}(1+B(\tilde{d}))\right] \\
\bar{B}=\bar{C} c\left[\tilde{r}\left(B(\tilde{d})+p_{0} C(|d|)+p_{1} B(\tilde{d})\right)\right]+ \\
+\left(B_{0}\left(d_{0}\right)+p_{0} B(\tilde{d})\right)\left(1+\frac{2 \bar{C}}{\kappa}\right)+s_{1} \bar{C}\left[1+\frac{2}{\kappa}(1+B(\tilde{d}))\right] .
\end{gathered}
$$

Assumption $\mathbf{H}[\boldsymbol{c}, \boldsymbol{d}, \boldsymbol{p}]$. Suppose that the constants $c, d=\left(d_{0}, d_{1}, d_{2}\right), p=\left(p_{0}\right.$, $\left.p_{1}\right)$ satisfy the conditions

$$
\begin{gathered}
d_{1}=p_{0} \geq s_{1}+c B(\tilde{d})\left(1+p_{0} r_{0}\right), \\
d_{2}=p_{1} \geq \max \left\{B(\tilde{d})\left(1+p_{0} r_{0}\right), \bar{A}, \bar{B}\right\}, \\
d_{0} \geq s_{0}+c\left[B_{0}\left(d_{0}\right)+B(\tilde{d}) p_{0}\right] .
\end{gathered}
$$

It follows from the continuity of $B_{0}, B, C \in \Theta$ that there are $d, p$ and sufficiently small $c \in(0, a]$ satisfying Assumption $\mathrm{H}[c, d, p]$.

Lemma 3. If $\varphi \in C^{1, L}[s]$ and Assumptions $\mathrm{H}[\alpha], \mathrm{H}[f], \mathrm{H}[c, d, p]$ are satisfied then $G^{(m)}: C_{\varphi, c}^{0, L}[p] \rightarrow C_{\varphi, c}^{0, L}[p]$.

Proof. Suppose that $u \in C_{\varphi, c}^{0, L}[p]$. Then

$$
\left\|G^{(m)}[u](t, x)\right\| \leq s_{1}+B(\tilde{d}) c\left(1+p_{0} r_{0}\right) \quad \text { on } \quad E_{c} .
$$

Now we prove that the function $G^{(m)}[u]$ satisfy the Lipschitz condition with constant $p_{1}$. If $(t, x),(\bar{t}, \bar{x}) \in E_{c}$ then

$$
\left\|G^{(m)}[u](t, x)-G^{(m)}[u](\bar{t}, \bar{x})\right\| \leq A_{\varphi}+A_{f},
$$

where

$$
\begin{gathered}
A_{\varphi}=\left\|\partial_{x} \varphi\left(Q\left[z^{(m)}, u\right](t, x)\right)-\partial_{x} \varphi\left(Q\left[z^{(m)}, u\right](\bar{t}, \bar{x})\right)\right\|, \\
A_{f}= \\
=\| \int_{\delta\left[z^{(m)}, u\right](t, x)}^{t}\left[\partial_{x} f\left(P\left[z^{(m)}, u\right](\tau, t, x)\right)+\partial_{w} f\left(P\left[z^{(m)}, u\right](\tau, t, x)\right) W^{(m)}\left[z^{(m)}, u\right](\tau, t, x)\right] d \tau- \\
-\int_{\delta\left[z^{(m)}, u\right](\bar{t}, \bar{x})}^{\bar{t}}\left[\partial_{x} f\left(P\left[z^{(m)}, u\right](\tau, \bar{t}, \bar{x})\right)+\partial_{w} f\left(P\left[z^{(m)}, u\right](\tau, \bar{t}, \bar{x})\right) W^{(m)}\left[z^{(m)}, u\right](\tau, \bar{t}, \bar{x})\right] d \tau \| .
\end{gathered}
$$

It follows from Lemmas 1, 2 that

$$
A_{\varphi} \leq s_{2} \bar{C}\left[1+\frac{2}{\kappa}(1+B(\tilde{d}))\right][|t-\bar{t}|+\|x-\bar{x}\|],
$$

and

$$
A_{f} \leq B(\tilde{d})\left(1+p_{0} r_{0}\right)|t-\bar{t}|+A_{0}[|t-\bar{t}|+\|x-\bar{x}\|] .
$$

ISSN 1027-3190. Укр. мат. журн., 2006, m. 58, № 6 
Then using Assumption $\mathrm{H}[c, d, p]$ we get

$$
\left\|G^{(m)}[u](t, x)-G^{(m)}[u](\bar{t}, \bar{x})\right\| \leq p_{1}[|t-\bar{t}|+\|x-\bar{x}\|]
$$

on $E_{c}$. This inequality together with (27) imply Lemma 3.

Lemma 4. Suppose that $\varphi \in C^{1, L}[s]$ and Assumptions $\mathrm{H}[\alpha], \mathrm{H}[f], \mathrm{H}[c, d$, $p]$ are satisfied. Then there exists exactly one function $\bar{u} \in C_{\varphi, c}^{0, L}[p]$ satisfying the equation $u=G^{(m)}[u]$.

Proof. Lemma 3 shows that $G^{(m)}: C_{\varphi, c}^{0, L}[p] \rightarrow C_{\varphi, c}^{0, L}[p]$. It follows that there is $A>0$ such that for $u, \tilde{u} \in C_{\varphi, c}^{0, L}[p]$ we have

$$
\left\|G^{(m)}[u](t, x)-G^{(m)}[\tilde{u}](t, x)\right\| \leq A \int_{0}^{t}\|u-\tilde{u}\|_{(\tau)} d \tau .
$$

Now we define the norm in the space $C_{\varphi, c}^{0, L}[p]$ as follows

$$
\|u\|_{\lambda}=\max \left\{\|u(t, x)\| e^{-\lambda t}:(t, x) \in E_{c}\right\}
$$

where $\lambda>A$. It is easy to see that $\left(C_{\varphi, c}^{0, L}[p],\|\cdot\|_{\lambda}\right)$ is a Banach space. Now we prove that there exists $q \in[0,1)$ such that

$$
\left\|G^{(m)}[u]-G^{(m)}[\tilde{u}]\right\|_{\lambda} \leq q\|u-\tilde{u}\|_{\lambda} \quad \text { for } \quad u, \tilde{u} \in C_{\varphi, c}^{0, L}[p] .
$$

According to (28), we have

$$
\begin{gathered}
\left\|G^{(m)}[u](t, x)-G^{(m)}[\tilde{u}](t, x)\right\| \leq \\
\leq A \int_{0}^{t}\|u-\tilde{u}\|_{(\xi)} d \xi=A \int_{0}^{t}\|u-\tilde{u}\|_{(\xi)} e^{-\lambda \xi} e^{\lambda \xi} d \xi \leq \\
\leq A\|u-\tilde{u}\|_{\lambda} \int_{0}^{t} e^{\lambda \xi} d \xi=\frac{A}{\lambda}\|u-\tilde{u}\|_{\lambda}\left(e^{\lambda t}-1\right) \leq \frac{A}{\lambda}\|u-\tilde{u}\|_{\lambda} e^{\lambda t}
\end{gathered}
$$

for $(t, x) \in E_{c}$. Then we have

$$
\left\|G^{(m)}[u](t, x)-G^{(m)}[\tilde{u}](t, x)\right\| e^{-\lambda t} \leq \frac{A}{\lambda}\|u-\tilde{u}\|_{\lambda}, \quad(t, x) \in E_{c} .
$$

It follows that the estimate (29) holds with $q=A \lambda^{-1}$. By the Banach fixed point theorem there exists exactly one $\bar{u} \in C_{\varphi, c}^{0, L}[p]$ satisfying the equation $u=G^{(m)}[u]$. This completes the proof of Lemma 4.

Suppose that $\varphi \in C^{1, L}[s]$. Let us denote by $T_{\varphi}$ the set of all functions $\omega: E^{*} \rightarrow R$ such that

(i) $\omega$ is continuous,

(ii) $\left.\omega\right|_{E_{0} \cup \partial_{0} E}=\varphi$.

Write $\mathcal{T}=\left\{i: d_{i}>0\right\}$. The following compatibility condition for the problem (1), (2) will be needed in our considerations.

Assumption $\mathbf{H}_{c}[f, \varphi]$. Suppose that

1) if $\omega, \tilde{\omega} \in T_{\varphi}$ then

$$
f\left(t, x, \omega_{\alpha(t, x)}, q\right)=f\left(t, x, \tilde{\omega}_{\alpha(t, x)}, q\right)
$$

for $x \in \Delta, q \in R^{n}$ and for almost all $t \in[0, a]$ 
2) there is a function $\psi: \partial_{0} E \rightarrow R^{n}, \psi=\left(\psi_{1}, \ldots, \psi_{n}\right)$, such that $\partial_{t} \varphi(t, x)=f\left(t, x, \tilde{\varphi}_{\alpha(t, x)}, \psi(t, x)\right)$ for $x \in \Delta$ and for almost all $t \in[0, a]$, where $\tilde{\varphi} \in T_{\varphi}$ and $\psi_{i}(t, x)=\partial_{x_{i}} \varphi(t, x)$ for $(t, x) \in[0, a] \times \Delta_{i}^{+} \cup \Delta_{i}^{-}, \quad i \in \mathcal{T}$.

Remark 3. Relation (30) can be considered as an assumption on $\varphi$ for $i \in \mathcal{T}$ and $t \in[0, c], \quad x \in \Delta_{i}^{+} \cup \Delta_{i}^{-}$and (30) is the equation for $\psi_{i}(t, x)$ if $i \in\{1, \ldots, n\} \backslash \mathcal{T}$, $t \in[0, c], x \in \Delta_{i}^{+} \cup \Delta_{i}^{-}$.

Remark 4. Suppose that $h_{0}=0, h=0$ and $F: E \times R \times R^{n} \rightarrow R$ is a given function and $f$ is defined by (3). Then (1) is equivalent to (4). If

$$
\alpha_{i}(t, x)=b_{i} \text { for } x \in \Delta_{i}^{+} \quad \text { and } \quad \alpha_{i}(t, x)=-b_{i} \text { for } x \in \Delta_{i}^{-},
$$

where $1 \leq i \leq n$ then condition 1 of the Assumption $\mathrm{H}_{c}[f, \varphi]$ is satisfied. For the above given $F$, consider the operator $f$ given by (6) and the differential integral equation with deviated variables (7). If

$$
\beta_{i}(t, x) \geq b_{i} \quad \text { for } \quad x \in \Delta_{i}^{+} \quad \text { and } \quad \beta_{i}(t, x) \leq-b_{i} \text { for } x \in \Delta_{i}^{-},
$$

where $1 \leq i \leq n$ then condition 1 of the Assumption $\mathrm{H}_{c}[f, \varphi]$ is satisfied. If $f$ is defined by (9) then we have differential integral equation (10). If

$$
\beta_{i}(t, x), \gamma_{i}(t, x) \geq b_{i} \text { for } x \in \Delta_{i}^{+} \quad \text { and } \beta_{i}(t, x), \gamma_{i}(t, x) \leq-b_{i} \text { for } x \in \Delta_{i}^{-} \text {, }
$$

where $1 \leq i \leq n$ then condition 1 of the Assumption $\mathrm{H}_{c}[f, \varphi]$ is satisfied.

Now we prove the main lemma in this section.

Lemma 5. If Assumptions $\mathrm{H}[\alpha], \mathrm{H}[f], \mathrm{H}[c, d, p], \mathrm{H}_{c}[f, \varphi]$ are satisfied, then for any $m \geq 0$ we have

$$
\partial_{x} z^{(m)}(t, x)=u^{(m)}(t, x) \quad \text { on } \quad E_{c}
$$

and $z^{(m)} \in C_{\varphi, c}^{1, L}[d]$.

Proof. We prove (31) by induction. It follows from the definition of the sequence $\left\{z^{(m)}, u^{(m)}\right\}$ that (31) is satisfied for $m=0$. Supposed now that condition (31) hold for a given $m \geq 0$, we will prove that the function $z^{(m+1)}$ given by (26) satisfies (31). Put

$$
\Delta^{(m)}(t, x, \bar{x})=z^{(m+1)}(t, \bar{x})-z^{(m+1)}(t, x)-u^{(m+1)}(t, x) \circ(\bar{x}-x),
$$

where $(t, x),(t, \bar{x}) \in E_{c}$. We prove that there exists $\tilde{C} \in R_{+}$such that

$$
|\Delta(t, x, \bar{x})| \leq \tilde{C}\|x-\bar{x}\|^{2} \quad \text { on } \quad E_{c} .
$$

It follows from (25), (26) that

$$
\begin{gathered}
\Delta^{(m)}(t, x, \bar{x})= \\
=F\left[z^{(m)}, u^{(m+1)}\right](t, \bar{x})-F\left[z^{(m)}, u^{(m+1)}\right](t, x)-G^{(m)}\left[u^{(m+1)}\right](t, x) \circ(\bar{x}-x) .
\end{gathered}
$$

Write

$$
\begin{aligned}
g^{(m)}(\tau, t, x)= & g\left[z^{(m)}, u^{(m+1)}\right](\tau, t, x), \quad \delta^{(m)}(t, x)=\delta\left[z^{(m)}, u^{(m+1)}\right](t, x), \\
& S^{(m)}(t, x)=\left(\delta^{(m)}(t, x), g^{(m)}\left(\delta^{(m)}(t, x), t, x\right)\right)
\end{aligned}
$$

and 


$$
Q^{(m)}(s, \tau, t, x, \bar{x})=s P\left[z^{(m)}, u^{(m+1)}\right](\tau, t, \bar{x})+(1-s) P\left[z^{(m)}, u^{(m+1)}\right](\tau, t, x) .
$$

Suppose that $\delta^{(m)}(t, \bar{x}) \leq \delta^{(m)}(t, x)$. Similar arguments apply to the case $\delta^{(m)}(t, \bar{x})>$ $>\delta^{(m)}(t, x)$. For simplicity of formulation of properties of the function $\Delta^{(m)}$ we define

$$
\begin{gathered}
A^{(m)}(t, x, \bar{x})=\varphi\left(S^{(m)}(t, \bar{x})\right)-\varphi\left(S^{(m)}(t, x)\right)- \\
-\partial_{x} \varphi\left(S^{(m)}(t, x)\right) \circ\left[g^{(m)}\left(\delta^{(m)}(t, \bar{x}), t, \bar{x}\right)-g^{(m)}\left(\delta^{(m)}(t, x), t, x\right)\right]- \\
-\partial_{t} \varphi\left(S^{(m)}(t, x)\right)\left[\delta^{(m)}(t, \bar{x})-\delta^{(m)}(t, x)\right]
\end{gathered}
$$

and

$$
\begin{gathered}
B^{(m)}(t, x, \bar{x})=\partial_{t} \varphi\left(S^{(m)}(t, x)\right)\left[\delta^{(m)}(t, \bar{x})-\delta^{(m)}(t, x)\right]+ \\
+\partial_{x} \varphi\left(S^{(m)}(t, x)\right) \circ\left[g^{(m)}\left(\delta^{(m)}(t, \bar{x}), t, \bar{x}\right)-g^{(m)}\left(\delta^{(m)}(t, x), t, x\right)-(\bar{x}-x)\right], \\
C^{(m)}(\tau, t, x, \bar{x})=\int_{\tau}^{t}\left[\partial_{q} f\left(P\left[z^{(m)}, u^{(m+1)}\right](\xi, t, \bar{x})\right)-\partial_{q} f\left(P\left[z^{(m)}, u^{(m+1)}\right](\xi, t, x)\right)\right] d \xi,
\end{gathered}
$$

and

$$
C^{(m)}=\left(C_{1}^{(m)}, \ldots, C_{n}^{(m)}\right) .
$$

Moreover we put

$$
\begin{gathered}
\Lambda^{(m)}(t, x, \bar{x})=\int_{\delta^{(m)}(t, \bar{x})}^{\delta^{(m)}(t, x)} f\left(P\left[z^{(m)}, u^{(m+1)}\right](\tau, t, \bar{x})\right) d \tau- \\
-\int_{\delta^{(m)}(t, \bar{x})}^{\delta^{(m)}(t, x)} \partial_{q} f\left(P\left[z^{(m)}, u^{(m+1)}\right](\tau, t, \bar{x})\right) \circ u^{(m+1)}\left(\tau, g^{(m)}(\tau, t, \bar{x})\right) d \tau .
\end{gathered}
$$

It follows from (34) that

$$
\begin{gathered}
\Delta^{(m)}(t, x, \bar{x})=\varphi\left(S^{(m)}(t, \bar{x})\right)-\varphi\left(S^{(m)}(t, x)\right)+ \\
+\int_{\delta^{(m)}(t, x)}^{t}\left[f\left(P\left[z^{(m)}, u^{(m+1)}\right](\tau, t, \bar{x})\right)-f\left(P\left[z^{(m)}, u^{(m+1)}\right](\tau, t, x)\right)\right] d \tau- \\
-\int_{\delta^{(m)}(t, x)}^{t} \partial_{q} f\left(P\left[z^{(m)}, u^{(m+1)}\right](\tau, t, \bar{x})\right) \circ u^{(m+1)}\left(\tau, g^{(m)}(\tau, t, \bar{x})\right) d \tau+ \\
+\int_{\delta^{(m)}(t, x)}^{t} \partial_{q} f\left(P\left[z^{(m)}, u^{(m+1)}\right](\tau, t, x)\right) \circ u^{(m+1)}\left(\tau, g^{(m)}(\tau, t, x)\right) d \tau+ \\
+\Lambda^{(m)}(t, x, \bar{x})-G^{(m)}\left[u^{(m+1)}\right](t, x) \circ(\bar{x}-x) .
\end{gathered}
$$

Having disposed this preliminary step, we apply the Hadamard mean value theorem to the difference

$$
f\left(P\left[z^{(m)}, u^{(m+1)}\right](\tau, t, \bar{x})\right)-f\left(P\left[z^{(m)}, u^{(m+1)}\right](\tau, t, x)\right) .
$$

We thus get 


$$
\Delta^{(m)}(t, x, \bar{x})=\bar{\Delta}^{(m)}(t, x, \bar{x})+\tilde{\Delta}^{(m)}(t, x, \bar{x}),
$$

where

$$
\begin{gathered}
\bar{\Delta}(t, x, \bar{x})=A^{(m)}(t, x, \bar{x})+ \\
+\int_{\delta^{(m)}(t, x)}^{t} \int_{0}^{1}\left[\partial_{x} f\left(Q^{(m)}(s, \tau, t, x, \bar{x})\right)-\right. \\
\left.-\partial_{x} f\left(P\left[z^{(m)}, u^{(m+1)}\right](\tau, t, x)\right)\right] d s \circ\left[g^{(m)}(\tau, t, \bar{x})-g^{(m)}(\tau, t, x)\right] d \tau+ \\
+\int_{\delta^{(m)}(t, x)}^{t} \int_{0}^{1}\left[\partial_{w} f\left(Q^{(m)}(s, \tau, t, x, \bar{x})\right)-\right. \\
\left.-\partial_{w} f\left(P\left[z^{(m)}, u^{(m+1)}\right](\tau, t, x)\right)\right] d s\left[z_{\alpha\left(\tau, g^{(m)}(\tau, t, \bar{x})\right)}-z_{\alpha\left(\tau, g^{(m)}(\tau, t, x)\right)}^{(m)}\right] d \tau+ \\
\left.+\int_{\delta^{(m)}(t, x) 0}^{t} \int_{\left.\left(P\left[z^{(m)}, u^{(m+1)}\right](\tau, t, \bar{x})\right)\right] d s \circ\left[\partial_{q} f\left(Q^{(m)}(s, \tau, t, x, \bar{x})\right)-\right.}^{1(m+1)}\left(\tau, g^{(m)}(\tau, t, \bar{x})\right)-u^{(m+1)}\left(\tau, g^{(m)}(\tau, t, x)\right)\right] d \tau+ \\
+\int_{\delta^{(m)}(t, x)}^{\partial_{w} f\left(P\left[z^{(m)}, u^{(m+1)}\right](\tau, t, x)\right)\left[z_{\alpha\left(\tau, g^{(m)}(\tau, t, \bar{x})\right)}-z_{\alpha\left(\tau, g^{(m)}(\tau, t, x)\right)}^{(m)}-\right.} \\
\left.-W^{(m)}\left[z^{(m)}, u^{(m+1)}\right](\tau, t, x) \circ\left(g^{(m)}(\tau, t, \bar{x})-g^{(m)}(\tau, t, x)\right)\right] d \tau
\end{gathered}
$$

and

$$
\begin{gathered}
\tilde{\Delta}^{(m)}(t, x, \bar{x})=B^{(m)}(t, x, \bar{x})+ \\
+\int_{\delta^{(m)}(t, x)}^{t} \partial_{x} f\left(P\left[z^{(m)}, u^{(m+1)}\right](\tau, t, x)\right) \circ\left[g^{(m)}(\tau, t, \bar{x})-g^{(m)}(\tau, t, x)-(\bar{x}-x)\right] d \tau+ \\
+\int_{\delta^{(m)}(t, x)}^{t} \partial_{w} f\left(P\left[z^{(m)}, u^{(m+1)}\right](\tau, t, x)\right) \times \\
\times\left[W^{(m)}\left[z^{(m)}, u^{(m+1)}\right](\tau, t, x) \circ\left[g^{(m)}(\tau, t, \bar{x})-g^{(m)}(\tau, t, x)-(\bar{x}-x)\right]\right] d \tau- \\
-\int_{\delta^{(m)}(t, x)}^{t}\left[\partial_{q} f\left(P\left[z^{(m)}, u^{(m+1)}\right](\tau, t, \bar{x})\right)-\right. \\
\left.-\partial_{q} f\left(P\left[z^{(m)}, u^{(m+1)}\right](\tau, t, x)\right)\right] \circ u^{(m+1)}\left(\tau, g^{(m)}(\tau, t, x)\right) d \tau+\Lambda^{(m)}(t, x, \bar{x}) .
\end{gathered}
$$

We will write an estimate for $\left|\tilde{\Delta}^{(m)}(t, x, \bar{x})\right|$. Since $g^{(m)}(\cdot, t, x)$ satisfies (14), we have

$$
g^{(m)}(\tau, t, \bar{x})-g^{(m)}(\tau, t, x)-(\bar{x}-x)=C^{(m)}(\tau, t, x, \bar{x}) .
$$

Substituting the above relation into $\tilde{\Delta}^{(m)}(t, x, \bar{x})$ and changing the order of integtals where necessary we obtain 


$$
\begin{gathered}
\tilde{\Delta}^{(m)}(t, x, \bar{x})=D^{(m)}(t, x, \bar{x})+ \\
+\int_{\delta^{(m)}(t, x)}^{t}\left[\partial_{q} f\left(P\left[z^{(m)}, u^{(m+1)}\right](\tau, t, \bar{x})\right)-\partial_{q} f\left(P\left[z^{(m)}, u^{(m+1)}\right](\tau, t, x)\right)\right] \circ E^{(m)}(\tau, t, x) d \tau,
\end{gathered}
$$

where

$$
\begin{gathered}
D^{(m)}(t, x, \bar{x})=\int_{\delta^{(m)}(t, \bar{x})}^{\delta^{(m)}(t, x)}\left[f\left(P\left[z^{(m)}, u^{(m+1)}\right](\tau, t, \bar{x})\right)-\partial_{t} \varphi\left(S^{(m)}(t, x)\right)\right] d \tau+ \\
+\int_{\delta^{(m)}(t, \bar{x})}^{\delta^{(m)}(t, x)}\left[\partial_{x} \varphi\left(S^{(m)}(t, x)\right)-u^{(m+1)}\left(\tau, g^{(m)}(\tau, t, \bar{x})\right)\right] \circ \partial_{q} f\left(P\left[z^{(m)}, u^{(m+1)}\right](\tau, t, \bar{x})\right) d \tau
\end{gathered}
$$

and

$$
\begin{gathered}
E^{(m)}(\tau, t, x)=-u^{(m+1)}\left(\tau, g^{(m)}(\tau, t, x)\right)+ \\
+\partial_{x} \varphi\left(S^{(m)}(t, x)\right)+\int_{\delta^{(m)}(t, x)}^{\tau}\left[\partial_{x} f\left(P\left[z^{(m)}, u^{(m+1)}\right](\xi, t, x)\right)+\right. \\
\left.+\partial_{w} f\left(P\left[z^{(m)}, u^{(m+1)}\right](\xi, t, x)\right) W^{(m)}\left[z^{(m)}, \bar{u}^{(m+1)}\right](\xi, t, x)\right] d \xi .
\end{gathered}
$$

The next claim is

$$
E^{(m)}(\tau, t, x)=0, \quad(t, x) \in E_{c}, \quad \tau \in I_{(t, x)} .
$$

We have

$$
g^{(m)}\left(s, \tau, g^{(m)}(\tau, t, x)\right)=g^{(m)}(s, t, x) \quad \text { and } \quad \delta^{(m)}\left(\tau, g^{(m)}(\tau, t, x)\right)=\delta^{(m)}(t, x)
$$

for $(t, x) \in E_{c}, \tau, s \in I_{(t, x)}$, therefore we get by (25)

$$
\begin{gathered}
u^{(m+1)}\left(\tau, g^{(m)}(\tau, t, x)\right)=\partial_{x} \varphi\left(\delta^{(m)}(t, x), g^{(m)}\left(\delta^{(m)}(t, x), t, x\right)\right)+ \\
+\int_{\delta^{(m)}(t, x)}^{\tau} \partial_{x} f\left(P\left[z^{(m)}, u^{(m+1)}\right](s, t, x)\right) d s+ \\
\left.+\int_{\delta^{(m)}(t, x)}^{\tau} \partial_{w} f\left(P\left[z^{(m)}, u^{(m+1)}\right](s, t, x)\right) W^{(m)}\left[z^{(m)}, \bar{u}^{(m+1)}\right](s, t, x)\right) d s
\end{gathered}
$$

for $(t, x) \in E_{c}, \quad \tau \in I_{(t, x)}$ and we have $E^{(m)}(\tau, t, x)=0$. Then we have proved that $\tilde{\Delta}^{(m)}(t, x, \bar{x})=D^{(m)}(t, x, \bar{x})$. We conclude from Assumption $\mathrm{H}_{c}[f, \varphi]$ and from Lemma 2, that there is $C_{1} \in R_{+}$such that

$$
\left|D^{(m)}(t, x, \bar{x})\right| \leq C_{1}\|x-\bar{x}\|^{2} \quad \text { on } \quad E_{c}
$$

and consequently

$$
\left|\tilde{\Delta}^{(m)}(t, x, \bar{x})\right| \leq C_{1}\|x-\bar{x}\|^{2} \quad \text { on } \quad E_{c} .
$$

Now we will write an estimate for $\left|\bar{\Delta}^{(m)}(t, x, \bar{x})\right|$. It follows from Assumptions $\mathrm{H}\left[\partial_{q} f\right], \mathrm{H}[f]$ that the terms

$$
\left\|\partial_{x} f\left(Q^{(m)}(s, \tau, t, x, \bar{x})\right)-\partial_{x} f\left(P\left[z^{(m)}, u^{(m+1)}\right](\tau, t, x)\right)\right\|,
$$




$$
\begin{gathered}
\left\|\partial_{q} f\left(Q^{(m)}(s, \tau, t, x, \bar{x})\right)-\partial_{q} f\left(P\left[z^{(m)}, u^{(m+1)}\right](\tau, t, \bar{x})\right)\right\|, \\
\left\|\partial_{w} f\left(Q^{(m)}(s, \tau, t, x, \bar{x})\right)-\partial_{w} f\left(P\left[z^{(m)}, u^{(m+1)}\right](\tau, t, x)\right)\right\|_{\star},
\end{gathered}
$$

are bounded from above by

$$
C(|d|) \tilde{r}\left\|g^{(m)}(\tau, t, \bar{x})-g^{(m)}(\tau, t, x)\right\| .
$$

An easy computation shows that

$$
\left|A^{(m)}(t, x, \bar{x})\right| \leq s_{2}\left\|g^{(m)}\left(\delta^{(m)}(t, \bar{x}), t, \bar{x}\right)-g^{(m)}\left(\delta^{(m)}(t, x), t, x\right)\right\|^{2},
$$

and

$$
\begin{gathered}
\left\|z_{\alpha\left(\tau, g^{(m)}(\tau, t, \bar{x})\right)}^{(m)}-z_{\alpha\left(\tau, g^{(m)}(\tau, t, x)\right)}^{(m)}\right\|_{0} \leq r_{0} d_{1}\left\|g^{(m)}(\tau, t, \bar{x})-g^{(m)}(\tau, t, x)\right\|, \\
\left\|u^{(m+1)}\left(\tau, g^{(m)}(\tau, t, \bar{x})\right)-u^{(m+1)}\left(\tau, g^{(m)}(\tau, t, x)\right)\right\| \leq p_{1}\left\|g^{(m)}(\tau, t, \bar{x})-g^{(m)}(\tau, t, x)\right\| .
\end{gathered}
$$

Since

$$
\partial_{x} z^{(m)}(t, x)=u^{(m)}(t, x) \quad \text { on } \quad E_{c},
$$

we have

$$
\begin{aligned}
& \left\|z_{\alpha\left(\tau, g^{(m)}(\tau, t, \bar{x})\right)}-z_{\alpha\left(\tau, g^{(m)}(\tau, t, x)\right)}^{(m)}-W^{(m)}\left[z^{(m)}, u^{(m+1)}\right](\tau, t, x) \circ\left[g^{(m)}(\tau, t, \bar{x})-g^{(m)}(\tau, t, x)\right]\right\|_{0} \leq \\
& \leq\left[p_{0} r_{1}+p_{1} r_{0}^{2}\right]\left\|g^{(m)}(\tau, t, \bar{x})-g^{(m)}(\tau, t, x)\right\|^{2} .
\end{aligned}
$$

The above estimates and the definition of $\bar{\Delta}^{(m)}$ imply

$$
\begin{gathered}
\left|\bar{\Delta}^{(m)}(t, x, \bar{x})\right| \leq s_{2}\left\|g^{(m)}\left(\delta^{(m)}(t, \bar{x}), t, \bar{x}\right)-g^{(m)}\left(\delta^{(m)}(t, x), t, x\right)\right\|^{2}+ \\
+A^{*} \int_{\delta^{(m)}(t, x)}^{t}\left\|g^{(m)}(\tau, t, \bar{x})-g^{(m)}(\tau, t, x)\right\|^{2} d \tau,
\end{gathered}
$$

where $A^{*}=B(\tilde{d})\left[p_{0} r_{1}+p_{1} r_{0}^{2}\right]+C(|d|) \tilde{r}^{2}$. It follows from Lemmas 1, 2 and from (14) that

$$
\left\|g^{(m)}\left(\delta^{(m)}(t, \bar{x}), t, \bar{x}\right)-g^{(m)}\left(\delta^{(m)}(t, x), t, x\right)\right\| \leq\left[B(\tilde{d}) \frac{2 \bar{C}}{\kappa}+\bar{C}\right]\|\bar{x}-x\| .
$$

Then there exists $C_{2} \in R_{+}$such that

$$
\left|\bar{\Delta}^{(m)}(t, x, \bar{x})\right| \leq C_{2}\|\bar{x}-x\|^{2} .
$$

Adding inequalities (36) and (37), we get (33) and consequently

$$
\partial_{x} z^{(m+1)}(t, x)=u^{(m+1)}(t, x) \quad \text { on } \quad E_{c} .
$$

This completes the proof of (31).

Now we prove that $z^{(m+1)} \in C_{\varphi, c}^{1, L}[d]$. It is clear that $z^{(m+1)}$ is continuous on $E_{c}$ and $z^{(m+1)}=\varphi$ on $\left(E_{0} \cup \partial_{0} E\right) \cap\left(\left[-h_{0}, c\right] \times R^{n}\right)$. Moreover from (31) it follows that

$$
\left\|\partial_{x} z^{(m+1)}(t, x)\right\| \leq d_{1}
$$

and

ISSN 1027-3190. Укр. мат. журн., 2006, m. 58, № 6 


$$
\left\|\partial_{x} z^{(m+1)}(t, x)-\partial_{x} z^{(m+1)}(\bar{t}, \bar{x})\right\| \leq d_{2}[|t-\bar{t}|+\|x-\bar{x}\|]
$$

on $E_{c}^{*}$. Our assumptions imply the estimates

$$
\left|z^{(m+1)}(t, x)\right| \leq d_{0}, \quad\left|z^{(m+1)}(t, x)-z^{(m+1)}(\bar{t}, \bar{x})\right| \leq d_{2}|t-\bar{t}|
$$

where $(t, x),(\bar{t}, \bar{x}) \in E_{c}^{*}$. This completes the proof of Lemma 5 .

Now we prove that the sequences $\left\{z^{(m)}\right\}$ and $\left\{u^{(m)}\right\}$ are uniformly convergent on $E_{c}$ if the constant $c$ is sufficiently small.

Put

$$
\tilde{A}=\bar{A}+C(|d|)\left(1+p_{0} r_{0}\right), \quad \tilde{B}=\tilde{A}+r_{0} B(\tilde{d}), \quad \tilde{D}=\bar{B}+(1+B(\tilde{d})) \tilde{B} e^{\tilde{A} c} .
$$

Assumption $\mathbf{H}[c]$. Suppose that the Assumption $\mathrm{H}[c, d, p]$ is satisfied and $c$ is such small constant that $c \tilde{D}<1$.

Lemma 6. If Assumptions $\mathrm{H}[\alpha], \mathrm{H}[f], \mathrm{H}[c]$ are satisfied, then the sequences $\left\{z^{(m)}\right\}$ and $\left\{u^{(m)}\right\}$ are uniformly convergent on $E_{c}$.

Proof. For $t \in[0, c]$ and $m \geq 1$ we put

$$
Z^{(m)}(t)=\left\|z^{(m)}-z^{(m-1)}\right\|_{t}, \quad U^{(m)}(t)=\left\|u^{(m)}-u^{(m-1)}\right\|_{(t)} .
$$

We prove that for $t \in[0, c]$ we have

$$
U^{(m+1)}(t) \leq \tilde{B} e^{\tilde{A} c} \int_{0}^{t}\left[Z^{(m)}(\tau)+U^{(m)}(\tau)\right] d \tau .
$$

It follows from Lemmas 1, 2 that we have the estimates

$$
\left\|g^{(m)}(\tau, t, x)-g^{(m-1)}(\tau, t, x)\right\| \leq \bar{C}\left|\int_{t}^{\tau}\left[Z^{(m)}(\tau)+U^{(m)}(\tau)+U^{(m+1)}(\tau)\right] d \tau\right|
$$

and

$$
\left|\delta^{(m)}(t, x)-\delta^{(m-1)}(t, x)\right| \leq \frac{2 \bar{C}}{\kappa} \int_{0}^{t}\left[Z^{(m)}(\tau)+U^{(m)}(\tau)+U^{(m+1)}(\tau)\right] d \tau,
$$

where $(t, x) \in E_{c}, \tau \in[0, c]$. Then we obtain the integral inequality

$$
U^{(m+1)}(t) \leq \tilde{A} \int_{0}^{t} U^{(m+1)}(\tau) d \tau+\tilde{B} \int_{0}^{t}\left[Z^{(m)}(\tau)+U^{(m)}(\tau)\right] d \tau,
$$

where $t \in[0, c]$. The above estimate and Gronwall inequality imply inequality (38). An easy computation shows that for $t \in[0, c]$ we have

$$
Z^{(m+1)}(t) \leq \bar{B} \int_{0}^{t}\left[Z^{(m)}(\tau)+U^{(m)}(\tau)+U^{(m+1)}(\tau)\right] d \tau+B(\tilde{d}) \int_{0}^{t} U^{(m+1)}(\tau) d \tau
$$

and consequently

$$
Z^{(m+1)}(t) \leq\left(\bar{B}+B(\tilde{d}) \tilde{B} e^{\tilde{A} c}\right) \int_{0}^{t}\left[Z^{(m)}(\tau)+U^{(m)}(\tau)\right] d \tau, \quad t \in[0, c] .
$$

The above inequality and (38) imply 


$$
Z^{(m+1)}(t)+U^{(m+1)}(t) \leq \tilde{D} \int_{0}^{t}\left[Z^{(m)}(\tau)+U^{(m)}(\tau)\right] d \tau, \quad t \in[0, c],
$$

where $m \geq 1$.

It follows from Lemma 3 that

$$
Z^{(1)}+U^{(1)} \leq 2\left(p_{0}+d_{0}\right), \quad t \in[0, c] .
$$

Then the uniform convergence of the sequences $\left\{z^{(m)}\right\}$ and $\left\{u^{(m)}\right\}$ follows from (41) and from the condition $c \tilde{D}<1$.

This completes the proof of Lemma 6.

4. The existence of solutions of nonlinear mixed problems. We are able to state the main result on the existence of generalized solutions to problem (1), (2). For a function $\varphi \in C^{1, L}[s]$ and for a point $\tau \in[0, a]$ we put

$$
\begin{aligned}
\|\varphi\|_{\tau} & =\max \left\{|\varphi(t, x)|:(t, x) \in\left(E_{0} \cup \partial_{0} E\right) \cap\left(\left[-h_{0}, \tau\right] \times R^{n}\right)\right\}, \\
\left\|\partial_{x} \varphi\right\|_{\tau} & =\max \left\{\left|\partial_{x} \varphi(t, x)\right|:(t, x) \in\left(E_{0} \cup \partial_{0} E\right) \cap\left(\left[-h_{0}, \tau\right] \times R^{n}\right)\right\} .
\end{aligned}
$$

Theorem 1. If Assumptions $\mathrm{H}[\alpha], \mathrm{H}[f], \mathrm{H}_{c}[f, \varphi]$ and $\mathrm{H}[c]$ are satisfied then for every $\varphi \in C^{1, L}[s]$ there exists a solution $v: E_{c}^{*} \rightarrow R$ to problem (1), (2). Moreover $v \in C_{\varphi, c}^{1, L}[d]$ and $\partial_{x} v \in C_{\varphi, c}^{0, L}[p]$.

If $\bar{\varphi} \in C^{1, L}[s]$ and $\bar{v} \in C_{\bar{\varphi}, c}^{1, L}[d]$ is a solution of equation (1) with initial boundary condition $z(t, x)=\bar{\varphi}(t, x)$ on $E_{0} \cup \partial_{0} E$ then there is $\Lambda_{c} \in R_{+}$such that

$$
\|v-\bar{v}\|_{t}+\left\|\partial_{x} v-\partial_{x} \bar{v}\right\|_{(t)} \leq \Lambda_{c}\left[\|\varphi-\bar{\varphi}\|_{t}+\left\|\partial_{x} \varphi-\partial_{x} \bar{\varphi}\right\|_{t}\right], \quad 0 \leq t \leq c .
$$

Proof. It follows from Lemmas 5 and 6 that there is $v \in C_{\varphi, c}^{1, L}[d]$ such that

$$
v(t, x)=\lim _{m \rightarrow \infty} z^{(m)}(t, x), \quad \partial_{x} v(t, x)=\lim _{m \rightarrow \infty} u^{(m)}(t, x)
$$

uniformly on $E_{c}$. Thus we get

$$
\begin{gathered}
v(t, x)=F\left[v, \partial_{x} v\right](t, x), \\
\partial_{x} v(t, x)=G\left[v, \partial_{x} v\right](t, x),
\end{gathered}
$$

and

$$
g\left[v, \partial_{x} v\right](\tau, t, x)=x+\int_{\tau}^{t} \partial_{q} f\left(P\left[v, \partial_{x} v\right](\xi, t, x)\right) d \xi
$$

Moreover, the initial boundary conditions

$$
v=\varphi, \quad \partial_{x} v=\partial_{x} \varphi \quad \text { on } \quad\left(E_{0} \cup \partial_{0} E\right) \times\left(\left[-h_{0}, c\right] \times R^{n}\right)
$$

are satisfied. It follows from the above relations that $v$ is a generalized solution of problem (1), (2) on $E_{c}^{*}$. The proof of the above property of $v$ is similar to the proof of an edequate properties for initial or initial boundary-value problems considered in [9] and [10] (Chapter IV). Details are omitted.

Now we prove relation (42). The functions $\left(\bar{v}, \partial_{x} \bar{v}\right)$ satisfies integral functional equations (20), (21) and initial boundary conditions (22) with $\bar{\varphi}$ instead of $\varphi$. It follows easily that there are $\Lambda_{0}, \Lambda_{1} \in R_{+}$such that the integral inequality 


$$
\begin{gathered}
\|v-\bar{v}\|_{t}+\left\|\partial_{x} v-\partial_{x} \bar{v}\right\|_{(t)} \leq \\
\leq \Lambda_{0}\left[\|\varphi-\bar{\varphi}\|_{t}+\left\|\partial_{x} \varphi-\partial_{x} \bar{\varphi}\right\|_{t}\right]+\Lambda_{1} \int_{0}^{t}\left[\|v-\bar{v}\|_{\tau}+\left\|\partial_{x} v-\partial_{x} \bar{v}\right\|_{(\tau)}\right] d \tau, \quad 0 \leq t \leq c,
\end{gathered}
$$

is satisfied. Using the Gronwall inequality we obtain (42) with $\Lambda_{c}=\Lambda_{0} \exp \left(\Lambda_{1} c\right)$. This proves the theorem.

Suppose that $\beta_{0}:[0, a] \rightarrow R, \beta^{\prime}: E \rightarrow R^{n}$ are given functions and that conditions (8) are satisfied. We consider the operator $f$ defined by (6). In this case (1) is equivalent to the differential equation with deviated variables (7). Now we formulate our existence result for problem (7), (2).

Assumption $\mathbf{H}[\boldsymbol{F}]$. Suppose that the function $F: E \times R \times R^{n} \rightarrow R$ of the variables $(t, x, p, q)$ satisfies the conditions

1) $F(\cdot, x, p, q):[0, a] \rightarrow R$ is measurable for each $(x, p, q) \in[-b, b] \times R \times R^{n}$ and there is $B_{0} \in \Theta$ such that

$$
|F(t, x, p, q)| \leq B_{0}(|p|) \quad \text { on } \quad E \times R \times R^{n} ;
$$

2) the partial derivatives

$$
\begin{gathered}
\left(\partial_{x_{1}} F(Q), \ldots, \partial_{x_{n}} F(Q)\right)=\partial_{x} F(Q), \quad Q=(t, x, p, q), \\
\left(\partial_{p_{1}} F(Q), \ldots, \partial_{p_{n}} F(Q)\right)=\partial_{p} F(Q), \\
\left(\partial_{q_{1}} F(Q), \ldots, \partial_{q_{n}} F(Q)\right)=\partial_{q} F(Q)
\end{gathered}
$$

exist for $(x, p, q) \in[-b, b] \times R \times R^{n}$ and for almost all $t \in[0, a]$;

3) the functions

$$
\begin{gathered}
\partial_{x} F(\cdot, x, p, q):[0, a] \rightarrow R^{n}, \quad \partial_{p} F(\cdot, x, p, q):[0, a] \rightarrow R, \\
\partial_{q} F(\cdot, x, p, q):[0, a] \rightarrow R^{n}
\end{gathered}
$$

are measurable and there is $\bar{B} \in R_{+}$such that

$$
\left\|\partial_{x} F(t, x, p, q)\right\| \leq \bar{B}, \quad\left\|\partial_{p} F(t, x, p, q)\right\| \leq \bar{B}, \quad\left\|\partial_{q} F(t, x, p, q)\right\| \leq \bar{B}
$$

for $(x, p, q) \in[-b, b] \times R \times R^{n}$ and for almost all $t \in[0, a]$;

4) there is $\bar{C} \in R_{+}$such that the functions $\partial_{x} F, \partial_{p} F, \partial_{q} F$ satisfies the Lipschitz condition with respect to $(x, p, q) \in[-b, b] \times R \times R^{n}$ for almost all $t \in[0, a]$.

Assumption $\mathbf{H}[\boldsymbol{\beta}]$. Suppose that the functions $\beta_{0}:[0, a] \rightarrow R, \beta^{\prime}: E \rightarrow R^{n}$ are such that

1) $0 \leq \beta_{0}(t) \leq t$ for $t \in[0, a]$ and $\beta^{\prime}(t, x) \in[-\bar{c}, \bar{c}]$ for $(t, x) \in E$ and for each $i, 1 \leq i \leq n$, and $t \in[0, a]$ we have

$$
\beta_{i}(t, x) \geq b_{i} \quad \text { for } \quad x \in \Delta_{i}^{+} \quad \text { and } \quad \beta_{i}(t, x) \leq b_{i} \text { for } x \in \Delta_{i}^{-} ;
$$

2) there is $\bar{r}_{0} \in R_{+}$such that

$$
\left|\beta_{0}(t)-\beta_{0}(\bar{t})\right| \leq \bar{r}_{0}|t-\bar{t}| \quad \text { on } \quad[0, a] ;
$$

3) $\beta^{\prime}$ is of class $C^{1}$ and $\left\|\partial_{x} \beta^{\prime}(t, x)\right\| \leq \bar{r}_{0}$ on $E$;

4) there is $\bar{r}_{1} \in R_{+}$such that 


$$
\left\|\partial_{x} \beta^{\prime}(t, x)-\partial_{x} \beta^{\prime}(\bar{t}, \bar{x})\right\| \leq \bar{r}_{1}[|t-\bar{t}|+\|x-\bar{x}\|] \quad \text { on } E .
$$

Theorem 2. Suppose that Assumptions $\mathrm{H}[\alpha], \mathrm{H}[F], \mathrm{H}[\beta]$ are satisfied and $\varphi \in C^{1, L}[s]$ and there is $\psi: \partial_{0} E \rightarrow R^{n}, \psi=\left(\psi_{1}, \ldots, \psi_{n}\right)$, such that

$$
\partial_{t} \varphi(t, x)=F(t, x, \varphi(\beta(t, x)), \psi(t, x))
$$

for $x \in \Delta$ and for almost all $t \in[0, a]$.

Then there is $c \in(0, a]$ and $v: E_{c}^{*} \rightarrow R$ such that $v$ is a solution of (7), (2) and $v \in C_{\varphi, c}^{1, L}[d], \partial_{x} v \in C_{\varphi, c}^{0, L}[p]$.

If $\bar{\varphi} \in C^{1, L}[s]$ and condition (43) is satisfied with $\bar{\varphi}$ instead of $\varphi$ and $\bar{v} \in C_{\bar{\varphi}, c}^{1, L}[d]$ is a solution of equation (7) with initial boundary condition $z(t, x)=$ $=\bar{\varphi}(t, x)$ on $E_{0} \cup \partial_{0} E$ then there is $\Lambda_{c} \in R_{+}$such that estimate (42) is satisfied.

Proof. Write

$$
\tilde{\beta}_{0}(t)=\beta_{0}(t)-\alpha_{0}(t), \quad \tilde{\beta}^{\prime}(t, x)=\beta^{\prime}(t, x)-\alpha^{\prime}(t, x), \quad(t, x) \in E,
$$

and $\tilde{\beta}(t, x)=\left(\tilde{\beta}_{0}(t, x), \tilde{\beta}^{\prime}(t, x)\right)$. Then the operator $f$ is defined by

$$
f(t, x, w, q)=F(t, x, w(\tilde{\beta}(t, x)), q), \quad(t, x, w, q) \in \Omega,
$$

and

$$
\begin{gathered}
\partial_{x} f(t, x, w, q)=\partial_{x} F(t, x, w(\tilde{\beta}(t, x)), q)+ \\
+\partial_{p} F(t, x, w(\tilde{\beta}(t, x)), q) \partial_{x} w(\tilde{\beta}(t, x)) \partial_{x} \tilde{\beta}^{\prime}(t, x), \\
\partial_{q} f(t, x, w, q)=\partial_{q} F(t, x, w(\tilde{\beta}(t, x)), q), \\
\partial_{w} f(t, x, w, q) \bar{w}=F(t, x, w(\tilde{\beta}(t, x)), q) \bar{w}(\tilde{\beta}(t, x)),
\end{gathered}
$$

where $(t, x, q) \in[0, a] \times[-b, b] \times R^{n}$ and $w \in C^{0,1}(D, R), \bar{w} \in C(D, R)$. It is clear that

$$
\begin{gathered}
\left\|\partial_{x} f(t, x, w, q)\right\| \leq \bar{B}\left[1+\left(r_{0}+\bar{r}_{0}\right)\|w\|_{1}\right], \\
\left\|\partial_{q} f(t, x, w, q)\right\| \leq \bar{B}, \quad\left\|\partial_{w} f(t, x, w, q)\right\|_{\star} \leq \bar{B} .
\end{gathered}
$$

We conclude from Assumptions $\mathrm{H}[f]$ and $\mathrm{H}[\alpha], \mathrm{H}[\beta]$ that

$$
\begin{gathered}
\left\|\partial_{x} f(t, \bar{x}, w+\bar{w}, \bar{q})-\partial_{x} f(t, x, w, q)\right\| \leq \\
\leq\left[\bar{C}\left[1+\left(r_{0}+\bar{r}_{0}\right)\|w\|_{1, L}\right]^{2}+\bar{B}\left[r_{1}+\bar{r}_{1}+\left(r_{0}+\bar{r}_{0}\right)^{2}\right]\|w\|_{1, L}\right]\|x-\bar{x}\|+ \\
+\bar{C}\left[1+\left(r_{0}+\bar{r}_{0}\right)\|w\|_{1, L}\right]\|q-\bar{q}\|+\left[\bar{c}+\left(r_{0}+\bar{r}_{0}\right)\left(\bar{B}+\bar{C}\|w\|_{1, L}\right)\right]\|w\|_{1}
\end{gathered}
$$

and

$$
\begin{aligned}
\left\|\partial_{q} f(t, \bar{x}, w+\bar{w}, \bar{q})-\partial_{q} f(t, x, w, q)\right\| & \leq \bar{C}\left[\left(1+\left(r_{0}+\bar{r}_{0}\right)\|w\|_{1, L}\right)\|x-\bar{x}\|+\|q-\bar{q}\|+\|w\|_{1}\right], \\
\left\|\partial_{q} f(t, \bar{x}, w+\bar{w}, \bar{q})-\partial_{w} f(t, x, w, q)\right\|_{\star} & \leq \bar{C}\left[\left(1+\left(r_{0}+\bar{r}_{0}\right)\|w\|_{1, L}\right)\|x-\bar{x}\|+\|q-\bar{q}\|+\|w\|_{1}\right] .
\end{aligned}
$$

It follows that all the assumptions of Theorem 1 are satisfied and the assertion follows.

Remark 5. It is important in our considerations that we have assumed the local with respect to the functional variable Lipschitz condition for the derivatives $\partial_{x} f$, 
$\partial_{w} f, \partial_{q} f$ on some special function spaces. Let us consider the simplest assumption. Suppose that there is $L \in R_{+}$such that

$$
\left\|\partial_{x} f(t, x, w, q)-\partial_{x} f(t, \bar{x}, \bar{w}, \bar{q})\right\| \leq L\left[\|x-\bar{x}\|+\|w-\bar{w}\|_{0}+\|q-\bar{q}\|\right]
$$

and that suitable estimates for the derivatives $\partial_{w} f$ and $\partial_{q} f$ are satisfied. It is easy to see that our results are true under the above assumptions. Now we show that our formulation of the Lipschitz condition is important.

It is easy to see that the operator $f$ defined by (6) does not satisfy condition of the form (44). Then there is a class of nonlinear equations satisfying Assumption $\mathrm{H}[f]$ and do not satisfying conditions of type (44).

Remark 6. In the case when (1) is reduced to the integral functional equation (10), one can formulate adequate assumptions and prove the particular version of the existence theorem, as easily as it was done above for the problem (7), (2).

1. Abolina V. E., Myshkis A. D. Mixed problems for semilinear hyperbolic systems on a plane // Mat. Sb. (N.S.). - 1960. - 50. - P. 423 - 442.

2. Myshkis A. D., SlopakA. S. A mixed problem for systems of differential functional equations with partial derivatives and with operators of the Volterra type // Mat. Sb. (N.S.). - 1957. - 41. P. $239-256$.

3. Turo J. Global solvability of the mixed problem for first order functional partial differential equations // Ann. pol. math. - 1991. - 52, № 3. - P. 231 - 238.

4. Turo J. Mixed problems for quasilinear hyperbolic systems // Proc. Second World Congress of Nonlinear Analysis, Pt 4 (Athens, 1996). Nonlinear Anal. - 1997. - 30, № 4. - P. 2329 - 2340.

5. Człapiński $T$. On the mixed problem for quasilinear partial differential-functional equations of the first order // Z. anal. Anwendungen. - 1997. - 16, № 2. - S. 463 - 478.

6. Cinquini $S$. On hyperbolic systems of (nonlinear) partial differential equations in several independent variables // Ann. mat. pura ed appl. - 1979. - 120, № 4. - P. 201 - 214.

7. Cinquini Cibrario M. New research on systems of nonlinear partial differential equations in several independent variables // Rend. Semin. mat. e fis. Milano. - 1982. - 52. - P. $531-550$.

8. Cinquini Cibrario M. A class of systems of nonlinear partial differential equations in several independent variables // Ann. mat. pura ed appl. - 1985. - 140, № 4. - P. 223 - 253.

9. Człapiński T. On the mixed problem for hyperbolic partial differential-functional equations of the first order // Czech. Math. J. - 1999. - 49 (124), № 4. - P. 791 - 809.

10. Kamont Z. Hyperbolic functional differential inequalities and applications. - Dordrecht: Kluwer Acad. Publ., 1999.

11. Van T. D., Tsuji M., Duy N. T. S. The characteristic method and its generalization for first-order nonlinear partial differential equations. - London: Chapman and Hall / CRC Boca Raton, 2000.

12. Kamenskij G. A., Myshkis A. D. Mixed functional-differential equations // Nonlinear Anal. - 1998. - 34, № 2. - P. $283-297$.

13. Myshkis A. D. On the solvability of the initial boundary problem for mixed functional differential equation of retarded type // Funct. Different. Equat. - 2000. - 7, № 3-4. - P. 311 - 324.

14. Myshkis A. D. Mixed functional differential equations with continuous and piecewise continuous solutions // Ibid. - 2002. - 9, № 1-2. - P. 221 - 226.

15. Topolski $K$. On the uniqueness of viscosity solutions for first order partial differential functional equations // Ann. pol. math. - 1994. - 59, № 1. - P. 65 - 75.

16. Topolski $K$. Classical methods for viscosity solutions of differential-functional inequalities // Nonlinear World. - 1997. - 4, № 1. - P. 1 - 17.

Received 13.07.2004 